\title{
Natural Resource Modeling WILEY
}

\section{Optimal fishery with coastal catch}

\section{Dieter Grass $^{1}$ | Hannes Uecker ${ }^{2}$ (i) $\mid$ Thorsten Upmann ${ }^{3,4,5}$}

${ }^{1}$ ORCOS, Institute of Statistics and Mathematical Methods in Economics, Vienna University of Technology, Vienna, Austria

${ }^{2}$ Institut für Mathematik, Universität Oldenburg, Oldenburg, Germany

${ }^{3}$ Helmholtz-Institute for Functional Marine Biodiversity, Oldenburg University, AWI, Oldenburg, Germany

${ }^{4}$ Faculty of Business Administration and Economics, Bielefeld University, Bielefeld, Germany

${ }^{5}$ Alfred-Wegener-Institut

Helmholtz-Zentrum fur Polar- und

Meeresforschung, Bremerhaven,

Germany

\section{Correspondence}

Hannes Uecker, Institut für Mathematik, Universität Oldenburg, 26111 Oldenburg, Germany.

Email: hannes.uecker@uol.de

\begin{abstract}
In many spatial resource models, it is assumed that an agent is able to harvest the resource over the complete spatial domain. However, agents frequently only have access to a resource at particular locations at which a moving biomass, such as fish or game, may be caught or hunted. Here, we analyze an infinite time-horizon optimal control problem with boundary harvesting and (systems of) parabolic partial differential equations as state dynamics. We formally derive the associated canonical system, consisting of a forward-backward diffusion system with boundary controls, and numerically compute the canonical steady states and the optimal time-dependent paths, and their dependence on parameters. We start with some one-species fishing models, and then extend the analysis to a predator-prey model of the Lotka-Volterra type. The models are rather generic, and our methods are quite general, and thus should be applicable to large classes of structurally similar bioeconomic problems with boundary controls.
\end{abstract}

\section{Recommedations for Resource Managers}

- Just like ordinary differential equation-constrained (optimal) control problems and distributed partial differential equation (PDE) constrained control problems, boundary control problems with PDE state dynamics may be formally treated by the Pontryagin's 
maximum principle or canonical system formalism (state and adjoint PDEs).

- These problems may have multiple (locally) optimal solutions; a first overview of suitable choices can be obtained by identifying canonical steady states.

- The computation of canonical paths toward some optimal steady state yields temporal information about the optimal harvesting, possibly including waiting time behavior for the stock to recover from a low-stock initial state, and nonmonotonic (in time) harvesting efforts.

- Multispecies fishery models may lead to asymmetric effects; for instance, it may be optimal to capture a predator species to protect the prey, even for high costs and low market values of the predators.

\section{K E Y W O R D S}

bioeconomics, bistable model, infinite time horizon, optimal boundary control, optimal harvesting, Pontryagin's maximum principle, predator-prey model

\section{1 | INTRODUCTION}

Optimal control (OC) theory is an important tool to design optimal harvesting strategies in the management of natural resources. Much of the literature considers only the temporal dimension, see, for example, the monographs of Conrad and Clark (1987), Conrad (2010), and Clark (2010), but recent work captures the spatial dimension as well. Early spatial models feature discrete patches, where at each location of the resource, the stock evolves according to an ordinary differential equation (ODE). Migration of the biomass is then modeled as entry and exit of the biomass from one location to the other, see, for example, Sanchirico and Wilen (1999), Behringer and Upmann (2014), and the references therein. However, in many cases, the continuous process of migration is more adequately described by partial differential equations (PDEs) characterizing the spread or diffusion of the resource within the domain.

For the case of ODE-constrained OC problems, a main tool is Pontryagin's maximum principle providing first-order necessary optimality conditions (Pontryagin, Boltyanskii, Gamkrelidze, \& Mishchenko, 1962); see also Aniţa (2000), Lenhart and Workman (2007), Grass, Caulkins, Feichtinger, Tragler, and Behrens (2008), and Aniţa, Arnăutu, and Capasso (2011) for textbook expositions, including many examples related to natural resources. In bioeconomics, the objective often contains a discounted time integral

$$
J=\int_{0}^{T} \mathrm{e}^{-\rho t} J_{c}(v(t), u(t)) \mathrm{d} t
$$

where $\rho>0$ is a discount rate, $v(t) \in \mathbb{R}^{n}$ are the states of the system, $u(t) \in U \subset \mathbb{R}^{p}$ represents the controls, and $J_{c}$ is called the current value function. If $T=\infty$ in (1), then we have infinite time horizons, while for $T<\infty$ we have a finite time horizon. Also for PDE-constrained OC 
problems, a large theory has been developed (Hinze, Pinnau, Ulbrich, \& Ulbrich, 2009; Li \& Yong, 1995; Lions, 1971; Tröltzsch, 2009). Some maximum principles have been rigorously established in an abstract way, assuming a priori the existence of optimal solutions, but almost exclusively for the finite time-horizon case. See Section 3 for further comments and references.

In a number of bioeconomic and resource-economic papers (e.g., Baker, Diele, Marangi, Martiradonna, \& Ragni, 2018; Ding \& Lenhart, 2009; Fister \& Lenhart, 2006; Fister, 1997; Kelly, Xing, \& Lenhart, 2016; Lenhart, Liang, \& Protopopescu, 1999), the existence of OCs, and subsequently the validity of the Pontryagin's maximum principle, has been proven on a case by case basis using some minimizing sequence and compactness arguments (see also Aniţa et al., 2011, chapter 5; Lenhart \& Workman, 2007, chapter 25). However, all these studies consider steady problems or finite time horizons. More specifically, Fister (1997) considers spatially distributed harvesting of both the prey and the predator (on the full spatial domain) in a predator-prey diffusive model population. In Fister (2001), the economic agent is interested in harvesting the predator only, but the model also introduces a boundary control, which allows for controlling the migration of the populations across the boundary (e.g., a fence, mesh size of a net, filter). Similarly, the further studies referred to above consider OC in a bioeconomic timedependent diffusive setting. One common result of these studies is that for distributed harvesting, it may be (economically) advantageous to have zones of no or substantially reduced harvest ("marine reserves" in the context of fisheries). The population may then grow to a high level in the no-harvest zone, and the "spillover" by diffusion gives a higher yield than the one obtained from a uniform harvesting. See also Leung (1995) and Neubert (2003) for similar results for steady-state problems.

For the PDE case with infinite time horizons, Pontryagin-type optimality conditions have been proven for some linear PDE problems with linear-quadratic objectives, for example, Barucci and Gozzi (2001), Faggian (2004), Faggian and Grosset (2013), Boucekkine, Camacho, and Fabbri (2013), and Ballestra (2016). For genuinely nonlinear problems with diffusion, such conditions have been formally derived in Brock and Xepapadeas (2008). See also Xepapadeas (2010), Brock and Xepapadeas (2010), Uecker (2016), and Grass and Uecker (2017) for applications that in particular may lead to the emergence of spatial heterogeneity of the resource and the harvesting.

Here, we assume an infinite time horizon and a spatially distributed resource for which harvesting can only be done on the boundary of the spatial domain. This constraint may result from legal or physical restraints where the agent is not allowed or not able to harvest inside the habitat. For instance, often a substantial part of fishing is done at the shore or near the shore by artisanal fisheries; also, as fishing or hunting may be banned within protected areas, such as nature reserves, marine protected areas, game reserves, and so forth, fishing and hunting frequently happens at the boundaries of those protected areas, aiming at spillover to the nonprotected areas (Fogarty \& Murawski, 2004; Kellner, Tetreault, Gaines, \& Nisbet, 2007; McCauley et al., 2016). Moreover, harvesting may be further restricted to subsets of the boundary, for example, to certain places at the shore of a lake. Specifically, we consider classes of infinite time-horizon OC fishery problems with diffusion, boundary catch, and classical Cobb-Douglas production functions. We start with some one-species problems, and then extend the analysis to a two-species predator-prey model. We formally (i.e., a priori assuming the existence of OCs, see Section 3.3 for further comments) derive the so-called canonical systems, that is the necessary first-order optimality conditions. The associated stationary problems are systems of nonlinear elliptic PDEs, which, depending on the parameter regimes, may have multiple solutions, and to first obtain a comprehensive picture of these so-called 
canonical steady states, we use the continuation and bifurcation software pde2path (Uecker, Wetzel, \& Rademacher, 2014). In the second step, we compute canonical paths to canonical steady states and compare their values, and thus characterize policies to reach optimal steady states in a profit-maximizing way.

It turns out that for both classes of models, that is, the scalar one and the interacting species model, generally speaking, a "moderate harvesting policy" is optimal. This is quite intuitive, as excessive fishing leads to a drastic diminution of the stock and thus impairs the conditions for future yield. A moderate fishing activity forgoes present profits, but saves some of the stock for later growth and catch. However, some results of our analysis are quite intriguing already for one of our scalar models, distinguished by a bistable growth function. Here, the space of initial states is divided into three sets, namely "subthreshold" initial states from which we can only reach low-stock canonical steady states, "superthreshold" initial states from which we can only reach high-stock canonical steady states, and a rather small "intermediate" set from which we can reach both.

We complement our analysis by discussing the spatial distributions of the respective costates or shadow prices. Whereas in the one-species models, the shadow price of fish falls with the distance from the shore, this does not necessarily hold for the predator in the two-species case, even though the net market value of the predator species is positive. Moreover, the asymmetric interaction between both species gives asymmetric cost effects: Whereas an increase in the fishing cost of the prey results in an increase in both stocks, an increase in the fishing cost of the predator leads to a decrease in the stock of the prey. In particular, if fishing of the predator is relatively costly, its value may become negative, even though its market price exceeds the harvesting cost. Of course, here we leave the gap in that we apply the Pontryagin's maximum principle in a formal way. Still, we identify candidates for optimal solutions, and we believe that our analysis makes a methodological contribution beyond the specific insights into optimal boundary fishing: Our approach can be applied to other PDE problems with controls restricted to the boundary of the domain, and is rather independent of the functional forms of the state PDEs and the objective function.

To the best of our knowledge, such infinite time-horizon boundary harvesting problems have not yet been considered in the bioeconomic literature. In Fister (2001), a finite time-horizon problem is considered, and, additionally to distributed controls, a rather special boundary control which is not directly connected to harvesting, whereas Kellner et al. (2007) focus on steady states, and the boundary harvest is not strictly at the boundary. Moreover, both use simple quadratic expressions for the yields as functions of efforts and stocks.

The remainder of the article is organized as follows. In Section 2, we describe our models, and also provide a phase plane analysis of the constraints for the scalar stationary case. In Section 3, we formally derive the canonical system for the scalar case, discuss this formal derivation, and describe the basic numerical method for their solution. In Section 4, we present the results for the one-species models. Results of the two-species model are in Section 5, and in Section 6 , we conclude by discussing possible extensions and future research directions.

\section{PROBLEM SETUP}

\subsection{A class of scalar models}

Consider a fishery problem where harvesting (fishing) can be done on the boundary of some area, for example, a fisher catching fish from the shore. For simplicity, we consider a 
one-dimensional space represented by the interval $\Omega:=(0, l)$, with fishing only at location $x=0$, the position of the fisher. Let $v=v(x, t)$ be the biomass of fish at location $x \in \Omega$ at time $t \geq 0$. The catch depends on the available biomass of fish $v$ and on the harvesting effort $k$ of the fisher, who wants to maximize his profit. We specify the catch (or harvest) as a standard Cobb-Douglas function:

$$
h=h(v, k)=v^{\alpha} k^{1-\alpha}
$$

with $0<\alpha<1$. Let $p>0$ denote the market price of one unit of fish, and $c>0$ the (constant) per unit cost of harvesting effort. As fish is a nondurable good, the catch is offered at the market immediately when it is realized. Thus, we model the instantaneous profit from harvesting as

$$
J_{c}(v, k)=p h(v, k)-c k .
$$

The evolution of the stock is governed by the net growth of the biomass and movement of fish. Possible growth functions are

$$
\begin{gathered}
f(v)=f_{\operatorname{lin}}(v):=\delta-\beta v \quad(\text { linear }), \\
f(v)=f_{\log }(v):=v(\delta-\beta v) \quad(\text { logistic }), \\
f(v)=f_{\mathrm{bi}}(v):=-(v-\delta)(v-\beta)(v-1) \quad(\text { bistable }),
\end{gathered}
$$

with parameters $\delta, \beta>0$, respectively, $0 \leq \delta<\beta<1$ for $f_{\text {bi }}$. For $f=f_{\text {lin }}$, the ODE $\dot{v}=f(v)$ has the unique globally stable fixed point $v^{*}=\delta / \beta$. For $f=f_{\log }$, we again have the stable fixed point $v^{*}$, and, in addition, the unstable fixed point $v=0$. For our purposes, both models are quite similar: In particular, for each set of parameters, both have a unique canonical steady state (CSS; which refers to steady states of the canonical system [CS], see below), showing similar qualitative behavior. The main difference is that for $f_{\text {lin }}$, we can compute these CSSs semianalytically (see Section 4.1.1), which can also be used to validate the numerics.

For $f_{\text {bi }}$, we have the stable fixed points $v=\delta$ and $v=1$, and the unstable fixed point $v=\beta$. For $\delta=0, f_{\text {bi }}$ models critical depensation (see, e.g., Conrad \& Clark, 1987, p. 63; Da Lara \& Doyen, 2008, p. 18f), meaning that to grow the stock needs to be beyond the threshold $\beta$, for example, for mating reasons (Allee effect). The parameter $\delta>0$ could be used to describe some small constant external input, that is, $f_{\mathrm{bi}}(0)=\delta \beta>0$, but we rather see $\delta$ as a regularization parameter, because $h$ in (2) is not differentiable at $v=0$ or $k=0$, that is, $\lim _{v \rightarrow 0} \partial_{v} h\left(v, k_{0}\right)=\lim _{k \rightarrow 0} \partial_{k} h\left(v_{0}, k\right)=\infty$ for all $v_{0}, k_{0}>0$. In economics, this models infinite marginal values (Inada conditions) at low stocks/harvesting efforts. For (initially) large stocks, we can also work with $\delta=0$, but here we shall also be interested in the case of optimal harvesting of low initial stocks. For $\delta>0$ we can derive and solve the CS, and obtain well behaved (steady and time-dependent) solutions with low $v$. We can then a posteriori take the limit $\delta \rightarrow 0$, which yields a well-defined limit CS. Moreover, the (steady and time-dependent) solutions of the CS for $\delta>0$ converge to solutions of the limit CS, and although we do not have a rigorous proof, this strongly suggests that the (low-stock) solutions of the limit CS yield optimal solutions of the problem with $\delta=0$. Altogether, we find that $f=f_{\text {bi }}$ gives a much richer problem than $f_{\operatorname{lin}}$ and $f_{\log }$ do. 
The movement of fish is modeled as diffusion, that is, by a term proportional to $\Delta v$, where $\Delta$ denotes the Laplace operator with respect to $x .{ }^{1}$ Thus, the biomass of fish evolves according to the system of differential equations and boundary conditions (BC):

$$
\begin{gathered}
\partial_{t} v=-G_{1}(v):=D \Delta v+f(v) \text { in } \Omega \times \mathcal{T}, \\
\partial_{n} v(l, t)=0 \quad \text { in } \mathcal{T} \text { (zero flux at the right boundary), } \\
D \partial_{n} v(0, t)+g(v(0, t), k(t))=0 \quad \text { in } \mathcal{T} \text { (control-dependent flux at the left boundary), } \\
v(x, 0)=v_{0}(x) \text { in } \Omega,
\end{gathered}
$$

where $D$ is the diffusion coefficient, $n$ denotes the exterior normal to the boundary $\partial \Omega$. In the one-dimensional case, we could assume (by rescaling the domain) that $D=1$, but for conceptual clarity and generalizations we keep $D$. The zero-flux (or Neumann) BC at $x=l$ model that no fish can leave or enter. The harvesting at the left boundary of $\Omega$ gives the flux BC (5c): If $g>0$, then also $\partial_{x} v(0, t)=-\partial_{n} v(0, t)=(1 / D) g>0$, meaning that $x \mapsto v(x, t)$ is an increasing function. As $g$ is induced by the harvest $h$, we set

$$
g(v, k)=\gamma h(v, k)
$$

for some $\gamma>0$. Hence, a larger takeout of fish at $x=0$ increases the differential in stocks between $x=0$ and its (right) neighborhood $x>0$, and $\gamma$ can be thought of as the inverse of the replacement flux of fish: when $\gamma$ is high (low) a given amount of fishing leads to a large (small) differential in stocks near $x=0$, and this differential is due to a slow (fast) replacement of fish due to diffusion. Thus, in a simple setting $\gamma$ could be chosen proportional to $1 / D$, but we keep it as an independent parameter, essentially to possibly model special conditions for the replacement fluxes at the boundary.

Finally, given the instantaneous profit $J_{c}$ from (3), the fisher seeks to maximize the total discounted profits:

$$
V\left(v_{0}\right)=\max _{k \in C\left([0, \infty), \mathbb{R}^{+}\right)} J\left(v_{0}, k\right), \quad \text { where } J\left(v_{0}, k\right):=\int_{0}^{\infty} \mathrm{e}^{-\rho t} J_{c}(v(0, t), k(t)) \mathrm{d} t .
$$

We thus have an OC problem with the PDE constraints (5a)-(5e) and the boundary control $k:[0, \infty) \rightarrow[0, \infty)$. In particular, we do not impose an upper limit on the harvesting effort $k$. Instead, it turns out that $k$ is bounded above, and that the natural constraint $k \geq 0$ is automatically fulfilled, that is, never becomes active.

\subsection{Phase plane analysis of the steady-state constraint}

To get an intuition for the constraints in (5), we first sketch a phase plane analysis of the constraint in the stationary case, that is, for the scalar ODE

${ }^{1}$ Although we focus on the one-dimensional case $\Omega=(0, l)$, where $\Delta v=\partial_{x}^{2} v$, we use dimension-independent notation where suitable for the purpose of possible generalization. Note that $\partial_{n} v(0, t)=-\partial_{x} v(0, t)$ and $\partial_{n} v(l, t)=\partial_{x} v(l, t)$. 


$$
D v^{\prime \prime}+f(v)=0 \text {, }
$$

where, as a shorthand, we write $v^{\prime} \equiv \partial_{x} v$ and $v^{\prime \prime} \equiv \partial_{x}^{2} v$. Equivalently, we can write (6) as a spatial dynamics system

$$
v_{1}^{\prime}=v_{2}, \quad D v_{2}^{\prime}=-f\left(v_{1}\right)
$$

which is Hamiltonian with conserved energy $E\left(v, v^{\prime}\right)=(1 / 2) D v^{\prime 2}+F(v)$, with $F^{\prime}=f$, because $(\mathrm{d} / \mathrm{d} x) E\left(v(x), v^{\prime}(x)\right)=v^{\prime}(x)\left(D v^{\prime \prime}(x)+f(x)\right)=0$, and hence the orbits are level lines of $E$. For the specifications of $f$ given in (4), we obtain

$$
\begin{gathered}
F_{\operatorname{lin}}(v)=\delta v-\frac{\beta}{2} v^{2}, \quad F_{\log }=\frac{\delta}{2} v^{2}-\frac{\beta}{3} v^{3}, \\
F_{\mathrm{bi}}(v)=\delta \beta v-\frac{1}{2}(\beta+(1+\beta) \delta) v^{2}+\frac{1}{3}(1+\beta+\delta) v^{3}-\frac{1}{4} v^{4} .
\end{gathered}
$$

Clearly, for all three, $\left(v_{1}, v_{2}\right)=(1,0)$ is a saddle point of the energy. From the modeling, we know that we should only be interested in orbits of (6) with $v(x)>0$ for all $x \in(0, l)$, with $v^{\prime}(l)=0$, and with $v^{\prime}(0)>0$ because we want a positive takeout at $x=0$. Thus, the only relevant solutions of (6) are those which start at $x=0$ with $v(0)>0$ and $v^{\prime}(0)>0$.

Figure 1a,b illustrates that $f_{\text {lin }}$ and $f_{\log }$ behave rather similarly in the pertinent region of the phase plane, that is, northwest of the fixed point $(1,0)$, but $f_{\mathrm{bi}}$ in Figure $1 \mathrm{c}, \mathrm{d}$ is qualitatively different. The black orbit in Figure $1 \mathrm{c}$ is similar to those in Figure 1a,b, that is it starts northwest and "near" the fixed point $(1,0)$. Additionally, see Figure 1d, there are now orbits (blue example) that start at low $v$ and $v^{\prime}$ and end near $(1,0)$, and orbits (magenta example) that start left of the low fixed point $(\delta, 0)$. Now, an immediate question is: For a given model, $f_{\text {lin }}, f_{\log }$, and $f_{\mathrm{bi}}$, which of its steady states maximize $J_{c}$ in the set $\mathcal{S}$ of steady states? Moreover, we are

(a)

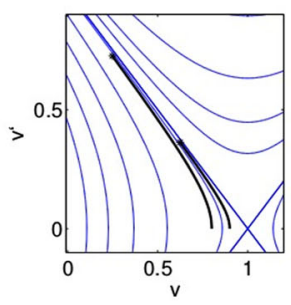

(b)

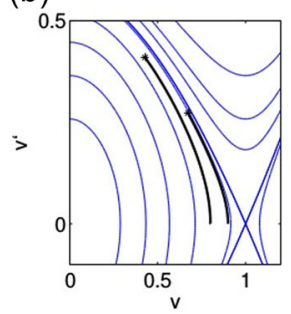

(c)

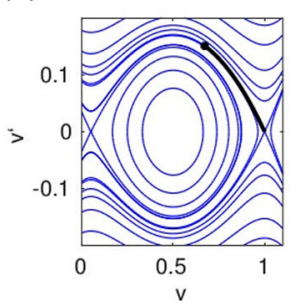

(d)

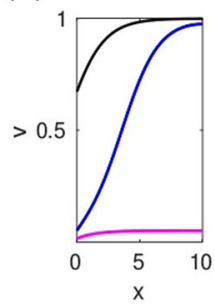

F I G U RE 1 Phase plane analysis for (7), with (a) $f=f_{\text {lin }}$ and (b) $f=f_{\log }$, both without loss of generality with $\delta=\beta=D=1$, and (c) $f=f_{\mathrm{bi}}$ with $\beta=0.5, \delta=0.05$. The blue lines are the level lines of the respective $E$, thus giving the phase portrait, while the black orbits (in (a,b)) are obtained from choosing $v(l)$ near $1, v^{\prime}(l)=0$, that is, $\left(v_{1}, v_{2}\right)(l)$ near the fixed point $(1,0)$, and integrating "backward in time" to $x=0$, here for illustration with $l=2$. The black points thus indicate the "initial condition" at $x=0$. We obtain qualitatively similar behavior for all $l>1$, say, with the only difference that for larger $l$, the "endpoints" of the black orbits must be closer to the fixed points in order that the solutions are in the first quadrant. In (c), we proceed similarly for $f_{\mathrm{bi}}$ with $l=10$. In (d), we plot $v(t)$ for three orbits; the black orbit is from (c), while the blue one corresponds to a slightly smaller $v(l)$, and the magenta one to nearly constant $v$ near the fixed point $\left(v, v^{\prime}\right)=(\delta, 0)$ 
interested in the solution of the intertemporal OC problem (5). Thus, in Section 3 we formally derive the necessary first-order optimality conditions, known as the CS, with steady solutions called CSSs. Returning to Figure 1 , for both, $f_{\text {lin }}$ and $f_{\text {log }}$, at a given set of parameters, there will be a unique CSS, which corresponds to one of the black orbits in Figure 1a,b, respectively. However, for $f=f_{\text {bi }}$ there will generically be multiple CSSs.

\section{3 | A predator-prey system}

The scalar model of Section 2.1 can be greatly generalized. As an example, consider a standard Lotka-Volterra system for prey $\left(v_{1}\right)$ and predator $\left(v_{2}\right)$ in the form

$$
\begin{aligned}
& \partial_{t} v_{1}=d_{1} \Delta v_{1}+\left(1-\beta v_{1}-v_{2}\right) v_{1}, \\
& \partial_{t} v_{2}=d_{2} \Delta v_{2}+\left(v_{1}-1\right) v_{2},
\end{aligned}
$$

with diffusion constants $d_{j}$, and self-damping parameter of the prey $\beta>0$. This system can more compactly be written as $\partial_{t} v=-G_{1}(v)=D \Delta v+f(v)$, with $D=\left(\begin{array}{cc}d_{1} & 0 \\ 0 & d_{2}\end{array}\right)$ and growth function $f(v)=\left(\begin{array}{c}\left(1-\beta v_{1}-v_{2}\right) v_{1} \\ \left(v_{1}-1\right) v_{2}\end{array}\right)$. Using the Liapunov function $\phi\left(v_{1}, v_{2}\right)=v_{1}+v_{2}-$ $\ln v_{1}-(1-\beta) \ln v_{2}$, it follows that

$$
V^{*}=\left(v_{1}, v_{2}\right)=(1,1-\beta)
$$

is the unique steady state of the ODE system $(\mathrm{d} / \mathrm{d} t) v=f(v)$ in the first quadrant, and is globally stable. Similarly, using the functional

$$
\Phi(t)=\int_{\Omega} \phi\left(v_{1}(t, x), v_{2}(t, x)\right) \mathrm{d} x,
$$

it follows that for $d_{1,2}$ sufficiently large, $V^{*}$ is the unique steady state of (10) with zero-flux $\mathrm{BC}$, and is globally stable (see, e.g., Hastings, 1978). For $v_{2} \equiv 0$, the $v_{1}$ equation corresponds to the case $f=f_{\log }$ from Section 2.1, and we have the additional fixed point $\left(v_{1}, v_{2}\right) \equiv(1 / \beta, 0)$, but here and in the following we always restrict to $v_{1}, v_{2}>0$.

Analogous to (5), we consider a boundary fishing problem for (10). We introduce $J$ and fishing efforts $k_{1}, k_{2}$ as controls via

$$
\begin{gathered}
J_{c}=\sum_{j=1}^{2} p_{j} h_{j}-c_{j} k_{j}, \quad h_{j}=h_{j}\left(v_{j}, k_{j}\right)=v_{j}^{\alpha_{j}} k_{j}^{1-\alpha_{j}}, \\
d_{j} \partial_{n} v_{j}=-g_{j}:=-\gamma_{j} h_{j} \quad \text { as left BC }
\end{gathered}
$$

and want to maximize

$$
V\left(v_{0}\right)=\max _{k \in C\left([0, \infty), \mathbb{R}_{+}^{2}\right)} J\left(v_{0}, k\right), \quad \text { subject to (10), (13) and (14), }
$$


where $J\left(v_{0}, k\right):=\int_{0}^{\infty} \mathrm{e}^{-\rho t} J_{c}(v(0, t), k(t)) \mathrm{d} t$, with the vector-valued initial states $v_{0}: \Omega \rightarrow \mathbb{R}_{+}^{2}$, and the vector-valued boundary control $k=\left(k_{1}, k_{2}\right):[0, \infty) \rightarrow \mathbb{R}_{+}^{2}$.

\section{3 | THE CS FORMALISM}

To solve (5), and (15), we formally derive the associated CSs, also called state-costate or state-adjoint equations, which here take the form of forward-backward diffusion problems. We first focus on the scalar case (5), and postpone the quite analogous vector-valued case (15) to Section 5. As already indicated in the Introduction, formally here inter alia means that we a priori assume that OCs exist. See Section 3.3 for further comments, and comparison with other methods and results for PDE-constrained infinite time-horizon OC.

\section{1 | Formal derivation}

We consider the Lagrangian

$$
L(v, \lambda, k):=\int_{0}^{\infty} \mathrm{e}^{-\rho t}\left\{J_{c}-\int_{\Omega} \lambda\left(\partial_{t} v+G_{1}(v)\right) \mathrm{d} x\right\} \mathrm{d} t
$$

where $\lambda: Q \rightarrow \mathbb{R}, Q:=\Omega \times[0, \infty)$ is the Lagrange multiplier for the PDE constraint (5a), also called the costate variable, or, in economics, the shadow price of the biomass at location $x$ at time $t$. Using integration by parts in $x$, we have

$$
\begin{aligned}
\int_{\Omega} \lambda D \Delta v \mathrm{~d} x & =-\int\langle\nabla \lambda, D \nabla v\rangle \mathrm{d} x+\int_{\partial \Omega} \lambda D \partial_{n} v \mathrm{~d} s \\
& =\int(D \Delta \lambda) v \mathrm{~d} x+\int_{\partial \Omega} \lambda\left(D \partial_{n} v\right)-\left(D \partial_{n} \lambda\right) v \mathrm{~d} s
\end{aligned}
$$

and integration by parts in $t$ yields

$$
-\int_{0}^{\infty} \mathrm{e}^{-\rho t} \int_{\Omega} \lambda \partial_{t} v \mathrm{~d} x \mathrm{~d} t=\int_{\Omega} \lambda(x, 0) v(x, 0) \mathrm{d} x+\int_{0}^{\infty} \mathrm{e}^{-\rho t} \int_{\Omega}\left(\partial_{t} \lambda-\rho \lambda\right) v \mathrm{~d} x \mathrm{~d} t
$$

where we used the so-called transversality condition

$$
\lim _{t \rightarrow \infty} \mathrm{e}^{-\rho t} \int_{\Omega} \lambda(x, t) v(x, t) \mathrm{d} x=0
$$

which here ensures that the boundary terms at $t=\infty$ vanish. See Section 3.3 for further comments. Next, using the BCs (5c) and (5b), that is, $\left.D \partial_{n} v\right|_{x=0}=-g$ and $\left.D \partial_{n} v\right|_{x=l}=0$, we obtain

$$
\begin{aligned}
L(v, \lambda, k)= & \int_{\Omega} v(x, 0) \lambda(x, 0) \mathrm{d} x+\int_{0}^{\infty} \mathrm{e}^{-\rho t}\left\{\left.\left(J_{c}-\lambda g-\left(D \partial_{n} \lambda\right) v\right)\right|_{x=0}-\left.\left(D \partial_{n} \lambda\right) v\right|_{x=l}\right. \\
& \left.-\int_{\Omega}\left(\rho \lambda-\partial_{t} \lambda-D \Delta \lambda\right) v-\lambda f(v) \mathrm{d} x\right\} \mathrm{d} t .
\end{aligned}
$$


The first variation of $L$ with respect to $v$, applied to a test function $\phi \in C^{\infty}(Q)$ with $\phi(\cdot, 0)=0$, yields

$$
\begin{aligned}
\partial_{v} L \phi= & \int_{0}^{\infty} \mathrm{e}^{-\rho t}\left\{\left.\left(\left(\partial_{v} J_{c}-\lambda \partial_{\nu} g-D \partial_{n} \lambda\right) \phi\right)\right|_{x=0}-\left.\left(D \partial_{n} \lambda\right) \phi\right|_{x=l}\right. \\
& \left.-\int_{\Omega}\left(\rho \lambda-\partial_{t} \lambda-D \Delta \lambda-\partial_{v} f(v) \lambda\right) \phi \mathrm{d} x\right\} \mathrm{d} t .
\end{aligned}
$$

Therefore, by density of $C^{\infty}(Q)$ in $L^{2}(Q)$, and by density of $\partial_{n} C^{\infty}(\Omega)$ in $L^{2}(\partial \Omega)$, the condition $\partial_{v} L \phi=0$ yields $\rho \lambda-\partial_{t} \lambda-D \Delta \lambda-\partial_{v} f(v) \lambda=0$ and the BCs $\left.D \partial_{n} \lambda\right|_{x=l}=0$ and $D \partial_{n} \lambda-\partial_{v} J_{c}+\lambda \partial_{\nu} g=0$. Thus, using $\partial_{v} J_{c}=p \partial_{v} h$ and $\partial_{v} g=\gamma \partial_{v} h$, the CS is

$$
\begin{gathered}
\partial_{t} v=D \Delta v+f(v), \quad v(x, 0)=v_{0}(x), \\
\partial_{t} \lambda=\rho \lambda-D \Delta \lambda-\partial_{v} f(v) \lambda, \\
D \partial_{n} v+\gamma h=0 \text { at } x=0, \quad \partial_{n} v=0 \text { at } x=l, \\
D \partial_{n} \lambda-(p-\gamma \lambda) \partial_{v} h=0 \text { at } x=0, \quad \partial_{n} \lambda=0 \text { at } x=l,
\end{gathered}
$$

where $k$ is obtained from $k(t)=\arg \max _{k} L(v(\cdot, t), \lambda(\cdot, t), k)$. In the absence of control constraints (see the remarks after (5f)), the condition $\partial_{k} L=0$ yields

$$
\begin{aligned}
0 & =\partial_{k} J_{c}-\gamma \lambda \partial_{k} h-v \partial_{k}(p-\gamma \lambda) \partial_{v} h \\
& =p \partial_{k} h-c-\lambda \gamma \partial_{k} h-v(p-\gamma \lambda) \partial_{v} \partial_{k} h=(p-\gamma \lambda) \partial_{k} h-(p-\gamma \lambda) \alpha \partial_{k} h-c,
\end{aligned}
$$

and thus

$$
(p-\gamma \lambda)(1-\alpha) \partial_{k} h=c \Leftrightarrow k=\tilde{k}(\lambda) \nu, \quad \tilde{k}(\lambda)=\left(\frac{(1-\alpha)^{2}(p-\gamma \lambda)}{c}\right)^{1 / \alpha}
$$

Hence, if an OC $k$ for the OC problem (5) exists, then (21) gives first-order optimality conditions. In particular, the optimal effort is determined by (21e), where $p-\gamma \lambda$ represents the total value of one unit of biomass. The extent to which a takeout affects future catch depends on the replacement flux of fish at the boundary, measured by $\gamma$. If $\gamma$ is large (small), then the replacement flux of fish is slow (fast), so that the stock recovers slowly (quickly). In economic terms, $\lambda$ is the shadow price of fish, and $\gamma \lambda$ represents a future reduction of profit due to today's takeout of fish, and this value must be subtracted from the market price of fish.

\subsection{Numerical method}

We want to solve (21) on the infinite time horizon $t \in[0, \infty)$, and thus at first might want to think of (21) as an initial value problem. However, (21a) provides initial data for only half the variables, while (21b) has backward diffusion. We proceed similar to Grass et al. (2008, chapter 7), Uecker (2016), and Grass and Uecker (2017) (see also Beyn, Pampel, \& Semmler, 2001; 
Kunkel and von dem Hagen, 2000). First, setting $u=(v, \lambda)$ we compute CSSs $\hat{u}$, that is, steady states of the CS. Then, given some initial state $v_{0}$, we want to compute canonical paths (CPs) $t \mapsto u(\cdot, t)=(v(\cdot, t), \lambda(\cdot, t))$ connecting $v_{0}$ to some CSS, and from these paths the OC $t \mapsto k(t)$ via (21e).

Thus, we write (21) as

$$
\partial_{t} u=-G(u), \quad u:=(v, \lambda), \quad u_{1}(x, 0)=v_{0}(x),
$$

where we generally suppress the dependence on parameters. The CSSs are solutions of

$$
G(u)=0 .
$$

In general, (23) is a nonlinear elliptic system, and thus at a given set of parameters we may expect multiple CSSs, $\hat{u}=(\hat{v}, \hat{\lambda})$, with (generically) different values $J(\hat{v})=(1 / \rho) J_{c}(\hat{v})$, for different CSSs. If, for instance, we let one parameter vary in (23); then the solutions come in branches (one-dimensional continua), from which at certain parameter values new solution branches may bifurcate. To find such solution branches, we use the Matlab continuation and bifurcation package pde2path (Uecker et al., 2014), designed for PDEs of type (22) (and also for more general PDEs) over one-, two- and threedimensional domains, based on a spatial finite-element method (FEM). As $u$ in (23) always has an even number of components, in the following, we write $2 n$ for the spatial degrees of freedom.

A CP connecting $v_{0}$ to some CSS $\hat{u}$ is a time-dependent solution $t \mapsto u(t)$ of (22) such that

$$
u_{1}(x, 0)=v_{0}(x) \text { and } \quad \lim _{t \rightarrow \infty} u(t)=\hat{u} .
$$

Only the first component $\left.u_{1}\right|_{t=0}=v_{0}$ is fixed, while $\left.u_{2}\right|_{t=0}=\left.\lambda\right|_{t=0}$ and hence the control $k(0)$ are free. Different situations may arise as follows:

1. There is a unique CP connecting $v_{0}$ to a CSS $\hat{u}$.

2. There is a unique CSS $\hat{u}$ which can be reached from $v_{0}$, but different CPs to do so.

3. Different CSSs $\hat{u}^{(1)}, \hat{u}^{(2)}, \ldots$ can be reached from $v_{0}$, and for each target there may be more than one CP.

If, given $v_{0}$, there is more than one $\mathrm{CP}$ by choice of $\lambda(0)$, then we can compare the respective values of $J$ for those paths, and decide which one is optimal. Conversely, we can also consider a given CSS $\hat{u}$ and ask from which $v_{0}$ it can be reached by a CP. In particular, a CSS $\hat{u}$ that can be reached from all nearby $v_{0}$ and such that the associated CPs maximizes $J\left(v_{0}, \cdot\right)$ is called a locally stable optimal steady state (OSS), whereas a CSS which can be reached from all $v_{0}$ that are admissible, that is, here all $v_{0} \geq 0$ (pointwise), and such that the CPs maximize $J\left(v_{0}, \cdot\right)$, is called a globally stable OSS. In general, all of the alternatives 1-3 can occur in a given system. In particular, there may be multiple locally stable OSSs. See, for example, Grass et al. (2008) for various ODE applications, and Uecker (2016) and Grass and Uecker (2017) for some PDE examples. Additionally, there may be optimal limit cycles (Uecker, 2019, section 3.4; Wirl, 1996), or (slow, i.e., such that (19) still holds) divergence of optimal solutions to infinity, see the AK example in Section 3.3.2. 
To compute a CP toward a CSS $\hat{u}$, we numerically proceed as follows. Given the spatial discretization of $G(u)=0$ with $2 n$ degrees of freedom, that is, $u=(v, \lambda) \in \mathbb{R}^{2 n}$, (22) becomes a coupled system of $2 n$ ODEs, which with a slight abuse of notation, we again write as

$$
M \frac{\mathrm{d}}{\mathrm{d} t} u=-G(u), \quad v(0)=v_{0} \in \mathbb{R}^{n}
$$

Here $M \in \mathbb{R}^{2 n \times 2 n}$ is the mass matrix of the FEM. We choose a truncation time $T$ and approximate (24) by

$$
u(T) \in E_{S}(\hat{u}) \text { and }\|u(T)-\hat{u}\|_{\infty} \text { small, }
$$

where $E_{S}(\hat{u})$ is the stable eigenspace of $\hat{u}$ for the linearization $M(\mathrm{~d} / \mathrm{d} t) \tilde{u}=-\partial_{u} G(\hat{u}) \tilde{u}$ of $(25)$. At $t=0$ we already have the BCs $v(0)=v_{0}$ for the states. Then, to obtain a well-defined two point boundary value problem in time we need

$$
\operatorname{dim} E_{S}(\hat{u})=n
$$

As the eigenvalues of the linearization are always symmetric around $\rho / 2$ (Grass \& Uecker, 2017, appendix A) we always have $\operatorname{dim} E_{S}(\hat{u}) \leq n$. The number

$$
d(\hat{u})=n-\operatorname{dim} E_{s}(\hat{u})
$$

is called the defect of the CSS $\hat{u}$. A CSS $\hat{u}$ with $d(\hat{u})>0$ is called defective, and if $d(\hat{u})=0$, then $\hat{u}$ has the so-called saddle point property (SPP). These are the only CSSs such that for general $v_{0}$ close to $\hat{v}$, we may expect a solution for the connecting orbits problem (25a) and (25b). See Grass and Uecker (2017) for further comments on the significance of the SPP (26) on the discrete level, and its (mesh-independent) meaning for the CS as a PDE. Furthermore, see Uecker (2017) for algorithmic details how to implement (25b) in pde2path, and how to find CPs connecting some $v_{0}$ to $\hat{u}$ by a continuation process in the initial states.

\subsection{Remarks on the formal derivation and comparison with other methods}

The derivation of (21) is formal in the sense that we assume

- that an OC $k:[0, \infty) \rightarrow \mathbb{R}$ exists and gives a finite value $J(k, v)<\infty$;

- that $k$ and the associated solution $v: \Omega \times \mathbb{R} \rightarrow \mathbb{R}$ are sufficiently smooth such that the integration by parts in $x$ and $t$ is valid;

- that the transversality condition (19) holds.

These are nontrivial assumptions. There are simple ODE OC problems where naive application of maximum principles yields the wrong results (see, e.g., Lenhart \& Workman, 2007, example 2.1, or Serovaiskii, 2003 for various further examples and a 
thorough discussion). ${ }^{2}$ Thus, it would be desirable to prove the existence of an OC $k$ for (5), and to prove that (21) yields all candidates. However, a general theory for OC for the for OC with PDE constraints so far only exists for finite time horizons (see, e.g., Casas \& Tröltzsch, 2015; Casas, Raymond, \& Zidani, 2000; Casas, Ryll, \& Tröltzsch, 2018; Raymond \& Zidani, 1999). The existing general theory for infinite time horizons only deals with ODEs, and usually the existence of an optimal solution is a priori assumed (see, e.g., Grass et al., 2008, sections 3.7, 3.8 and the references therein, or Tauchnitz, 2015).

In summary, (21) only gives some candidates for optimal solutions, that is, candidates within the class for which (21) can be derived, and the solutions we obtain are necessarily regular in $t$ and $x$. Thus, we cannot guarantee that there are no "reasonable" controls $k$, for example, $k \in P C^{0}\left([0, \infty), \mathbb{R}_{+}\right)$, that yield a higher $J$ than that obtained from the candidates from (21). Moreover, we did not consider state or control constraints. However, our aim are computations for models based on the Cobb-Douglas harvesting (2) that go beyond simple models based on linear-quadratic objective functionals (see Sections 3.3.1 and 3.3.2 below). In particular, we can check a posteriori that all CSSs and CPs fulfill the natural constraints $k(t)>0$ for all $t \geq 0$, and $v(x, t) \geq 0$ for all $t \geq 0$ and all $x \in \Omega$, and that both are also reasonably bounded from above. Thus, we think that the existence of controls yielding a higher objective value, not obtained via (21), is very unlikely.

On the other hand, there is some work which deals rigorously with (linear-quadratic) infinite time-horizon OC problems with linear PDE constraints. In the following two subsections, we give two examples and relate these to our formal analysis and the two-step numerical approach from Section 3.2.

\subsection{1 | Age-specific advertising}

In Faggian and Grosset (2013) optimal advertising models with a linear-quadratic objective are studied, for instance, of the form

$$
V\left(v_{0}\right)=\max _{w \in \mathcal{W}} J\left(v_{0}, w\right), \quad J\left(v_{0}, w\right)=\int_{0}^{\infty} \mathrm{e}^{-\rho t}\left(\int_{\Omega} \pi(x) v(x, t)-\frac{\kappa(x)}{2} w^{2}(x, t) \mathrm{d} x\right) \mathrm{d} t,
$$

subject to the first-order (transport) linear PDE:

$$
\partial_{t} v=-\partial_{x} v-\delta v+w \text { in } \Omega=(0, \omega), \quad v(0, t)=0, \quad v(x, 0)=v_{0}(x)
$$

Here, $[0, \omega]$ is an age interval of customers, $v:[0, \omega] \times[0, \infty) \rightarrow \mathbb{R}_{+}$is the (age and timedependent) goodwill of the customers with respect to some product, the control $w(x, t)$ is the advertising effort, distributed in age and time, and $\pi, \kappa: \Omega \rightarrow \mathbb{R}$ describe the profits and costs of advertising, respectively. Similar first-order PDE OC problems have also been considered in Barucci and Gozzi (2001) and Feichtinger, and Veliov (2003) (see also Grass et al., 2008, section 8.3). Using results based on Barucci and Gozzi (2001), Faggian and Grosset (2013) provide some rigorous analysis, which inter alia yields the following:

\footnotetext{
${ }^{2}$ An even simpler example would be the function $f: \mathbb{R} \rightarrow \mathbb{R}, f(x)=x^{3}$, which has a unique solution $x=0$ of the "necessary conditions" $f^{\prime}(x)=3 x^{2}=0$, but $f$ has no maximum or mimimum. Also, if we restrict $f$ to $I=[-1,1]$, then the extrema are at $x= \pm 1$ where $f^{\prime}(x)=0$ does not hold. Clearly, $f^{\prime}(x)=0$ is only necessary for inner extrema, and in any case is in general not sufficient.
} 
[A] Assume that $\pi, \kappa \in L^{2}(\Omega), v_{0} \in H^{1}\left(\Omega, \kappa>0, \pi, v_{0} \geq 0\right.$, and take

$$
\mathcal{W}=L_{\rho}^{p}([0, \infty), \mathbb{R})=\left\{w:[0, \infty) \rightarrow \mathbb{R}_{+}, \int_{0}^{\infty} \mathrm{e}^{-\rho t}\|w(t)\|_{L^{2}}^{p} \mathrm{~d} t\right\}
$$

as the set of admissible controls. Then (28) has a unique optimal advertising strategy $w^{*}=w^{*}(x)$, which in particular does not depend on $t$. The associated optimal solution $v^{*}: \Omega \times[0, \infty)$ is

$$
v^{*}(x, t)=\mathrm{e}^{-\delta t} v_{0}(x-t) \chi_{[t, \omega]}(x)+\int_{0}^{\min t, x} \mathrm{e}^{-\delta s} \frac{\bar{\pi}(x-s)}{\kappa(x-s)} \mathrm{d} s
$$

where $\chi_{I}$ is the characteristic function of the interval $I$, that is, $\chi_{I}(x)=1$ if $x \in I, 0$ else, and $\bar{\pi} \in H^{1}(\Omega)$ can be explicitly computed. In particular, for all $v_{0} \in H^{1}(\Omega), v^{*}(\cdot, t) \rightarrow \hat{v}(\cdot)$ as $t \rightarrow \infty$, where $\hat{v}(x)=\int_{0}^{x} \mathrm{e}^{-\delta s}((\bar{\pi}(x-s)) /(\kappa(x-s))) \mathrm{d} s$.

Similar results can be obtained for related (linear-quadratic) models, which may also include boundary controls (see, e.g., Barucci \& Gozzi, 2001; Faggian, 2004). The proofs use some ideas from dynamic programming, and a posteriori yield a Pontryagin maximum principle. Additionally, Faggian and Grosset (2013) provide and discuss some interesting explicit results for specific choices of $\pi$ and $\kappa$.

We obtain the same results from directly applying the CS formalism as follows. The Lagrangian reads

$$
L(v, \lambda, w):=\int_{0}^{\infty} \mathrm{e}^{-\rho t}\left\{\int_{0}^{\omega} \pi v-\frac{\kappa}{2} w^{2}-\lambda\left(\partial_{t} v+\partial_{x} v+\delta v-w\right) \mathrm{d} x\right\} \mathrm{d} t
$$

and by integration by parts in $t$ of $\int_{0}^{\infty} \mathrm{e}^{-\rho t} \int_{\Omega} \lambda \partial_{t} \nu \mathrm{d} x \mathrm{~d} t$ (using the TC (19)), and integration by parts in $x$ of $\int_{\Omega} \lambda \partial_{x} v \mathrm{~d} x$, and taking first variations wrt $\lambda$ and $v$ we obtain

$$
\begin{gathered}
\partial_{t} v=-\partial_{x} v-\delta v+w, \quad v(x, 0)=v_{0}(x), \quad v(0, t)=0, \\
\partial_{t} \lambda=-\partial_{x} \lambda+(\rho+\delta) \lambda-\pi, \quad \lambda(\omega, t)=0,
\end{gathered}
$$

while $\delta_{w} L=0$ yields $w=\lambda / \kappa$. Importantly, because $J_{c}$ has no mixed term of the form $v w$, (32b) decouples from (32a) and can thus be discussed first. This is also at the core of the solution via dynamic programming. Here, (32b) has a unique steady solution $\hat{\lambda}(x)$, which can be computed to be $\kappa(x) w^{*}(x)$. Moreover, (32b) is ill-posed as an initial value problem because the $\mathrm{BC}$ $\lambda(\omega, t)=0$ is on the "wrong side" as the transport in $(32 \mathrm{~b})$ is still to the right. Hence, $\hat{\lambda}(x)$ is the only feasible solution of (32b), and plugging $\hat{w}=\hat{\lambda} / \kappa$ back into (32a) we obtain (30). Moreover, concerning the (numerical) SPP in (26), the structure of (32a) and (32b), that is, the fact that (32b) is ill-posed and decoupled from (32a), yields the fact that every perturbation of $\hat{\lambda}$ is in the unstable eigenspace of $\hat{u}$, and hence a priori $\lambda \equiv \hat{\lambda}$ for every CP.

All this is readily confirmed by applying the numerics from Section 3.2 to (32a) and (32b). Additionally, for the numerics we can easily extend (28) and (29) beyond the linear-quadratic case. 


\subsection{2 | The spatial AK model}

A problem which is particularly interesting to illustrate the role of the TC (19), is the "spatial AK model” analyzed in Boucekkine et al. (2013) and Ballestra (2016). It reads

$$
V\left(v_{0}\right)=\max _{c \in C} J\left(v_{0}, c\right), \quad J\left(v_{0}, c\right)=\int_{0}^{\infty} \mathrm{e}^{-\rho t}\left(\int_{\Omega} \mathcal{U}(c(x, t)) \mathrm{d} x\right) \mathrm{d} t, \quad \mathcal{U}(c)=\frac{c^{1-\sigma}}{1-\sigma},
$$

subject to the linear diffusion equation

$$
\partial_{t} v=\partial_{x}^{2} v+A v-c(t, x) \text { in } \Omega=(0,2 \pi),\left.\quad v\right|_{t=0}=v_{0}
$$

with the periodic BC $v(0, t)=v(2 \pi, t), \partial_{x} v(0, t)=\partial_{x} v(2 \pi, t)$ (exactly the same results can be obtained for homogeneous Neumann $\left.\mathrm{BC} \partial_{n} v\right|_{\partial \Omega}=0$ over general domains). Here, $v=v(x, t)$ is a distribution of capital, the control $c=c(x, t)$ is consumption, and $A, \rho>0$ and $\sigma \in(0,1)$. In Boucekkine et al. (2013), the following result is shown via dynamic programming, and has been related to Pontryagin's maximum principle in Ballestra (2016):

[B] Assume that $A(1-\sigma)<\rho$, and let $\eta=(\rho-A(1-\sigma)) /(2 \pi \sigma), \beta=(A-\rho) / \sigma$ and $\bar{v}_{0}=$ $\int_{0}^{2 \pi} v_{0}(x) \mathrm{d} x$. Then $c^{*}=c^{*}(t)=c_{0} \mathrm{e}^{\beta t}$ with $c_{0}=\eta \bar{v}_{0}$ is the unique OC for (33).

In [B], the admissible controls fulfill $c, v \geq 0$ and a suitable limiting transversality condition, which fixes the constant $c_{0}$ in $c^{*}$, which is discussed in detail and expressed in various ways in Boucekkine et al. (2013) and Ballestra (2016).

Here, we want to illustrate that the CS formalism from Section 3.1 yields exactly the same result via the equivalent TC (19). The Lagrangian reads

$$
L(v, \lambda, c)=\int_{0}^{\infty} \mathrm{e}^{-\rho t}\left(\int_{\Omega} \mathcal{U}(c)-\lambda\left(\partial_{t} v-\partial_{x}^{2} v-A v+c\right) \mathrm{d} x\right) \mathrm{d} t
$$

and going through the integration by parts (using the periodic BC in $x$ and the TC (19) in $t$ ) and taking first variations, we obtain the CS:

$$
\begin{gathered}
\partial_{t} v=\partial_{x}^{2} v+A v-c, \quad c=\left(\frac{1}{\lambda}\right)^{1 / \sigma},\left.\quad v\right|_{t=0}=v_{0}, \\
\partial_{t} \lambda=-\partial_{x}^{2} \lambda+(\rho-A) \lambda,
\end{gathered}
$$

with periodic $\mathrm{BC}$ for $v$ and $\lambda$. Again, (35b) decouples, and has the general solution

$$
\lambda(x, t)=\sum_{n \in \mathbb{N}_{0}} \mathrm{e}^{\mu_{n} t}\left(a_{n} \cos (n x)+b_{n} \sin (n x)\right), \quad \mu_{n}=n^{2}+(\rho-A), \quad a_{n}, b_{n} \in \mathbb{N}, b_{0}=0,
$$

but the nonnegativity $\lambda>0$ (cause $c>0$ ) yields $a_{n}=b_{n}=0$ for all $n \in \mathbb{N}$. Thus, $\lambda(x, t)=\lambda(t)=\mathrm{e}^{(\rho-A) t} \lambda_{0}$ for some $\lambda_{0}>0$, and consequently 


$$
c(t)=\mathrm{e}^{(A-\rho) t / \sigma} c_{0} \quad \text { with } c_{0}=\left(1 / \lambda_{0}\right)^{1 / \sigma} .
$$

With $\mathrm{e}^{t \partial_{x}^{2}}$ the semigroup for the linear diffusion equation $\partial_{t} v=\partial_{x}^{2} v$ on $\Omega$, the solution $v(x, t)$ of (35a) then reads

$$
v(x, t)=\mathrm{e}^{t\left(\partial_{x}^{2}+A\right)} v_{0}-c_{0} \int_{0}^{t} \mathrm{e}^{(t-s) A} \mathrm{e}^{\beta s} \mathrm{~d} s=\mathrm{e}^{t\left(\partial_{x}^{2}+A\right)} v_{0}+c_{0} \mathrm{e}^{t A} \frac{1}{2 \pi \eta}\left(\mathrm{e}^{(1 / \sigma)(A(1-\sigma)-\rho) t}-1\right) .
$$

As $\mathrm{e}^{t \partial_{x}^{2}}$ (with Neumann BC or periodic BC) conserves the average $\bar{v}=\int_{\Omega} v(x) \mathrm{d} x$, we obtain

$$
\bar{v}(t)=\mathrm{e}^{t A}\left(\bar{v}_{0}+\frac{c_{0}}{\eta}\left(\mathrm{e}^{(A(1-\sigma)-\rho) t / \sigma}-1\right)\right) .
$$

For $A(1-\sigma)<\rho$ we have $\mathrm{e}^{(A(1-\sigma)-\rho) t / \sigma} \rightarrow 0$ for $t \rightarrow \infty$, and the TC

$$
\begin{aligned}
\lim _{t \rightarrow \infty} \mathrm{e}^{-\rho t} \int \lambda(t) v(x, t) \mathrm{d} x & =\lim _{t \rightarrow \infty} \mathrm{e}^{-\rho t} \lambda(t) \bar{v}(t) \\
& =\lim _{t \rightarrow \infty} \mathrm{e}^{(A-\rho) t} \lambda_{0} \mathrm{e}^{(\rho-A) t}\left(\bar{v}_{0}+\frac{c_{0}}{\eta}\left(\mathrm{e}^{(A(1-\sigma)-\rho) t / \sigma}-1\right)\right) \stackrel{!}{=} 0
\end{aligned}
$$

uniquely fixes $c_{0}=\eta \bar{v}_{0}$ in dependence of the initial mass, illustrating the role of (19). In particular, if one (wrongly) chooses $c_{0}>c_{0}^{*}$, then $\bar{v}(t) \rightarrow-\infty$ as $t \rightarrow \infty$, clearly violating the assumption $v(x, t) \geq 0$ for all $x, t$, and $\lim _{t \rightarrow \infty} \mathrm{e}^{-\rho t} \lambda(t) \bar{v}(t)<0$. Conversely, if $c_{0}<c_{0}^{*}$ then $\lim _{t \rightarrow \infty} \mathrm{e}^{-\rho t} \lambda(t) \bar{v}(t)>0$, and consumption is smaller than optimal.

Note that $\bar{v}(t), c(t) \rightarrow \infty$ if $\rho<A$ (long sighted consumers) and $\bar{v}(t), c(t) \rightarrow 0$ if $\rho>A$ (short sighted consumers), and in these cases the method from Section 3.2 does not apply. In the special case $\rho=A$, for which $c^{*}(t) \equiv c_{0}=\eta \bar{v}_{0}$, we have (for fixed parameter values) a line $\left\{(\hat{v}, \hat{\lambda}): \hat{v}>0, \hat{\lambda}=1 /(\eta \hat{v})^{\sigma}\right\}$ of spatially constant CSSs, parameterized by the mass $\hat{v}$. These are "weak" saddle points in the sense that one eigenvalue always equals zero, and given a nonnegative initial state $v_{0}=v_{0}(x)$ we obtain a unique CP to the CSS with $\hat{v}=\bar{v}_{0}$.

\section{4 | RESULTS FOR THE SCALAR FISHERY PROBLEMS}

We return to (5) and want to compute CSSs and CPs, starting with $f=f_{\text {lin }}$ and $f_{\log }$. Whereas, in general, we need numerics already for CSSs, for the case $f=f_{\text {lin }}$ in (5) we can also compute the CSSs (semi)analytically. This can be used to verify the numerics, and, via comparative statics, the modeling.

\section{1 | Linear growth and logistic growth}

\subsubsection{Semianalytical CSSs for linear growth}

For $f_{\text {lin }}(v)=\delta-\beta v$, the steady CS takes the form (without loss of generality choosing $D=1$ ):

$$
0=v^{\prime \prime}+\delta-\beta v, \quad 0=(\rho+\beta) \lambda-\lambda^{\prime \prime},
$$




$$
v^{\prime}(0)=\gamma h, \quad v^{\prime}(l)=0, \quad \lambda^{\prime}(0)=-(p-\gamma \lambda) \partial_{v} h, \quad \lambda^{\prime}(l)=0 .
$$

The ODEs in (37a) decouple, and the same holds true for the associated time-dependent PDEs, which makes them superficially similar to the examples in Section 3.3 but the two problems are still coupled by the BC at $x=0$ in (37b). The general solution of (37a) is

$$
v(x)=\delta / \beta+a_{1} \mathrm{e}^{\sqrt{\beta} x}+b_{1} \mathrm{e}^{-\sqrt{\beta} x}, \quad \lambda(x)=a_{2} \mathrm{e}^{r x}+b_{2} \mathrm{e}^{-r x}, \quad r=\sqrt{\rho+\beta},
$$

and it remains to compute the constants $a_{1}, a_{2}, b_{1}, b_{2}$ from the BCs in (37b). From the Neumann $\mathrm{BC}$ at $x=l$ we get

$$
b_{1}=a_{1} \mathrm{e}^{2 \sqrt{\beta} l}, \quad b_{2}=a_{2} \mathrm{e}^{2 r l},
$$

and thus end up with an algebraic system for $a_{1}, a_{2}$, namely

$$
\sqrt{\beta} a_{1}\left(\mathrm{e}^{2 \sqrt{\beta} l}-1\right)=\gamma k^{1-\alpha} v^{\alpha}, \quad r a_{2}\left(\mathrm{e}^{2 r l-1}\right)=\alpha k^{1-\alpha} v^{\alpha-1}(p-\gamma \lambda)
$$

together with $k=\left((1-\alpha)^{2}(p-\gamma \lambda) / c\right)^{1 / \alpha}$, where it is understood that $\lambda=\lambda(0)$ and $v=v(0)$ are functions of $a_{1}, a_{2}$ via (38) and (39).

We now give a bioeconomic discussion of the CSSs, aiming to illustrate general features. Letting

$$
\Psi(x, a):=\frac{\mathrm{e}^{x a}+\mathrm{e}^{a(2 l-x)}}{a\left(\mathrm{e}^{2 l a}-1\right)},
$$

the steady solutions of (37) can be implicitly written as

$$
v(x)=\delta / \beta-\gamma h(v(0), k) \Psi(x, \beta), \quad \lambda(x)=(p-\gamma \lambda(0)) \partial_{v} h(v(0), k) \Psi(x, r),
$$

with spatial dependence given by $\Psi$. The stock of the resource in the CSS equals its steady-state value $v^{*}=\delta / \beta$ minus some decrement, which depends on $x$ and the decay rate $\beta$, but does not (directly) depend on the discount rate $\rho$. As $\Psi$ is decreasing in $x$, the stock is higher and the shadow price is lower at more distant locations. Both results mirror the fact that a takeout of fish exclusively takes place at the boundary $x=0$. Due to the diffusion of fish, its removal at $x=0$ is compensated by gradual replenishment, but as this process takes time, the value of fish is lower at more distant locations.

System (40) cannot easily be solved algebraically for $a_{1,2}$, that is, for $v(0), \lambda(0)$ and $k$, but it can immediately and conveniently be solved numerically in Mat lab or similar systems. As an example, we choose the parameter specification

$$
(\rho, \alpha, p, \gamma, \beta, \delta, l)=(0.02,0.3,1,0.5,0.01,0.01,20)
$$

and let $c$ vary between 0.1 and 2 . For $c=0.1,1,2$, we then obtain the unique solutions $\left(a_{1}, a_{2}\right)=(-0.167,0.0014), \quad\left(a_{1}, a_{2}\right)=(-0.0077,0.00022), \quad$ and $\left(a_{1}, a_{2}\right)=(-0.0027,0.00006)$, 
(a) $c=0.1$

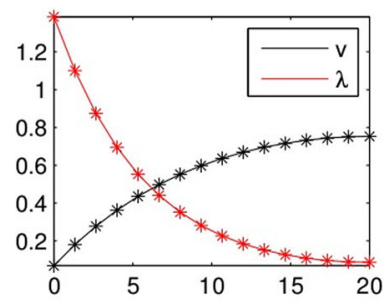

$c=1$

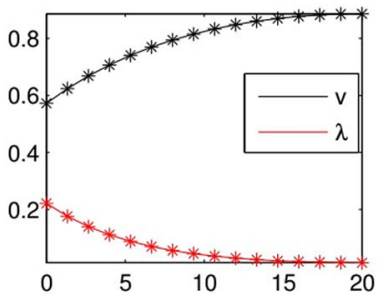

$c=2$

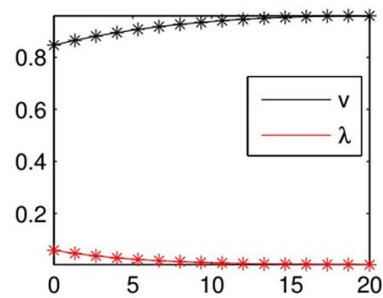

(b)
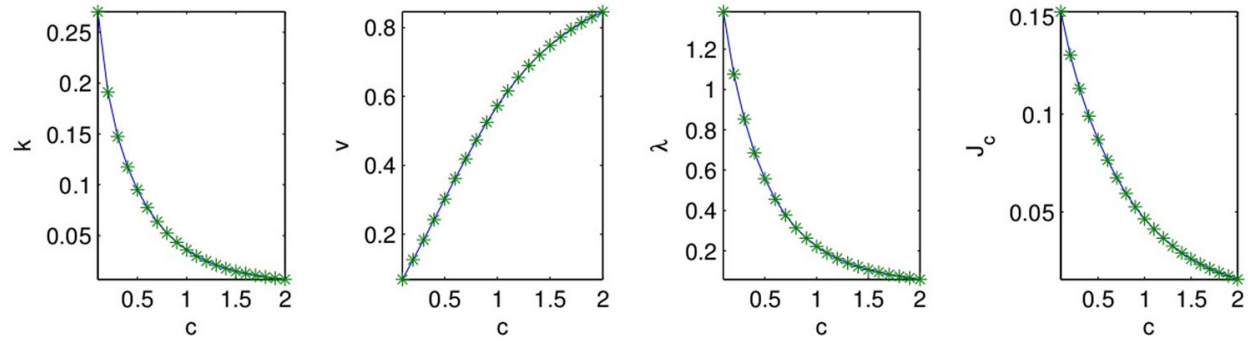

F I G U RE 2 Some results for linear growth. In (a) we compare the solution (38) from (40) with parameters (41) (dots) with the direct numerical solution of (37) with pde2path (lines). Similarly, (b) compares the dependence of $k, v, \lambda$, and $J_{c}$ on $c$, computed via the two approaches

respectively. The associated solutions (38) are shown in Figure 2a, and are indistinguishable from the direct numerical solution of (37) using pde2path.

Following Section 3.2, the next step would be to compute the time-dependent CPs from an arbitrary initial state $v_{0}$ (with $v_{0}(x)>0$ for all $x$ ) to the (for fixed parameters) unique CSS, which is always a saddle point, and the computation of their values. However, it turns out that the case $f=f_{\log }$ is quite similar to $f=f_{\text {lin }}$, and thus here we skip the presentation of CPs for $f=f_{\text {lin }}$ as we present CPs for $f=f_{\text {log }}$ in Section 4.1.3.

\subsection{2 | Comparative statics}

Figure $2 \mathrm{~b}$ illustrates the dependence of the economically interesting quantities $k, h, J_{c}$ and of $v(0), \lambda(0)$ on the effort $\operatorname{cost} c$. Their qualitative behavior can also be obtained analytically, which in economics is called comparative statics. From (21e) and (40), we obtain $v=v(0)$, $\lambda=\lambda(0)$ and $k$ as implicit functions of the cost parameter $c$. Using the shorthand $\Psi(a)=\Psi(0, a)$ we have

$$
v=\frac{\delta}{\beta}-\gamma h(v, k) \Psi(\sqrt{\beta}), \quad \lambda=(p-\gamma \lambda) \partial_{v} h(v, k) \Psi(r), \quad c=(p-\gamma \lambda)(1-\alpha) \partial_{k} h(v, k) .
$$

Implicitly differentiating (42) with respect to $c$, using $h(v, k)=v^{\alpha} k^{1-\alpha}$ and solving for the desired derivatives yields 


$$
\begin{gathered}
v^{\prime}(c)=\frac{v \gamma k^{\alpha+1} \Psi(\sqrt{\beta})\left(v k^{\alpha}+k \alpha \gamma v^{\alpha} \Psi(r)\right)}{(1-\alpha) \alpha(p-\gamma \lambda)\left(v k^{\alpha}+k \gamma v^{\alpha} \Psi(r)\right)\left(v k^{\alpha}+k \gamma v^{\alpha} \Psi(\sqrt{\beta})\right)}>0, \\
k^{\prime}(c)=-\frac{k^{\alpha+1} v^{-\alpha}\left(v k^{\alpha}+k \alpha \gamma v^{\alpha} \Psi(r)\right)\left(v k^{\alpha}+k \alpha \gamma v^{\alpha} \Psi(\sqrt{\beta})\right)}{(1-\alpha)^{2} \alpha(p-\gamma \lambda)\left(v k^{\alpha}+k \gamma v^{\alpha} \Psi(r)\right)\left(v k^{\alpha}+k \gamma v^{\alpha} \Psi(\sqrt{\beta})\right)}<0, \\
\lambda^{\prime}(c)=-\frac{k^{\alpha+1} \Psi(r)}{(1-\alpha)\left(v k^{\alpha}+k \gamma v^{\alpha} \Psi(r)\right)}<0, \\
h^{\prime}(c)=-\frac{v^{\prime}(c)}{\gamma \Psi(\sqrt{\beta})}<0 .
\end{gathered}
$$

An increase in $c$ renders harvesting effort more and more unattractive: Effort $k$ is reduced implying a reduction in harvest $h$, and thus an increase in the stock $v$; in parallel, higher costs reduce the value of the stock $\lambda$. Correspondingly, the resulting profit falls with higher values of $c$. As the comparative effects (43) are unambiguous, it is clear that our numerical results depicted in Figure $2 \mathrm{~b}$ are not due to our parameter specification but illustrate general features of our model.

\subsubsection{Logistic growth}

Similarly to the case $f=f_{\text {lin }}$, for $f=f_{\text {log }}$ we again have unique CSSs $(v(x), \lambda(x))$ which look qualitatively and even quantitatively similar to those for $f=f_{\text {lin }}$. The reason for this similarity is that $f(v)=v\left(\delta-\beta v\right.$ ) is positive in the relevant range $0<v<v^{*}=\delta / \beta$ (see also our discussion of the phase plane in Section 2.2). Accordingly, the dependence on parameters such as $c$ is also qualitatively similar for logistic growth and linear growth. Moreover, it turns out that the unique CSSs $\hat{u}$ for both models are globally stable OSSs, that is, for any $v_{0} \geq 0$ (pointwise, with $v_{0}(x)>0$ at least at one point) there is a $\mathrm{CP}$ connecting $v_{0}$ to $\hat{u}$. In Figure 3 we show two CPs for $f=f_{\log }$. In Figure $3 \mathrm{a}, \mathrm{b}$, we choose $v_{0} \equiv 1$ (full initial stock in the domain), and in Figure $3 \mathrm{c}, \mathrm{d}, v_{0} \equiv 0.1$ (almost depleted

(a)

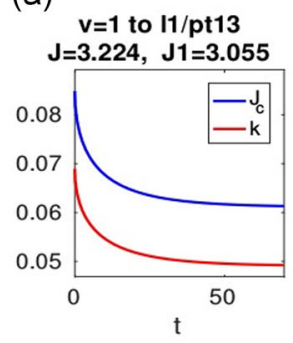

(b)

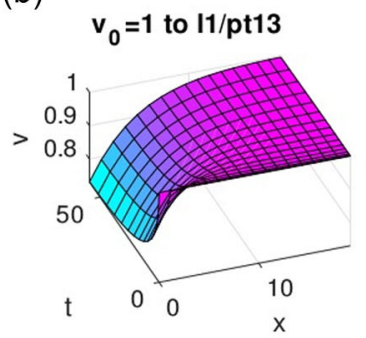

(c)

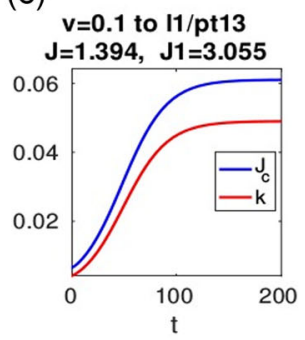

(d)

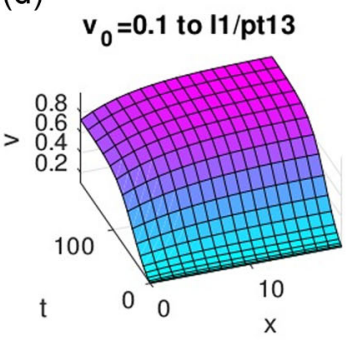

FI G URE 3 Example CPs connecting initial states $v_{0}$ to the unique globally stable CSS $\hat{u}$ for $f=f_{\log }$, $(\rho, \alpha, p, \gamma, \delta, \beta)=(0.02,0.3,1,0.5,0.01,0.01), c=1$, and $v_{0} \equiv 1$ in $(\mathrm{a}, \mathrm{b})$, and $v_{0} \equiv 0.1$ in (c,d). (a,c) show $k, J_{c}$, while (b,d) illustrate the associated evolution of $v$. Here, $J=\int_{0}^{\infty} \mathrm{e}^{-\rho t} J_{c}(u(t)) \mathrm{d} t$ and $J 1:=\int_{0}^{\infty} \mathrm{e}^{-\rho t} J_{c}(\hat{u}) \mathrm{d} t=(1 / \rho) J_{c}(\hat{u})$ denote the values of the CP and of the target CSS, respectively 
stock). Figure 3a,c shows the control $k$ and the current value $J_{c}$ at the left boundary as a function of time, while Figure $3 b, c$ shows the associated evolutions of $v{ }^{3}$ The results are as expected: Starting at a high (low) $v_{0}$, the fishing effort decreases (increases) monotonically to that of the CSS. The main benefit of such a numerical computation is to find the precise quantitative behavior of $t \mapsto k(t)$.

\section{2 | Bistable growth}

Compared with $f_{\text {lin }}$ and $f_{\log }$, for $f=f_{\text {bi }}$ there are some important differences: depending on $v(l)$ (with $v^{\prime}(l)=0$ ) we may expect CSSs with $v$ of the black, blue, and magenta type indicated in Figure $1 \mathrm{~d}$. We now fix $l=10$, and, in contrast to Figure 2 where we used the costs $c$ as continuation parameter, we fix $c$ and consider

$$
(\alpha, p, \beta, \gamma, c)=(0.2,8,0.5,1,1)
$$

as a base parameter set. We also first fix $\delta=0.05$, and start with the discount rate $\rho$ as our primary continuation parameter for the computation of (branches of) CSSs, because this turns out to be more convenient to obtain multiple CSSs. Subsequently we study the dependence of "low-stock CSSs" $\hat{u}(\cdot ; \delta)$ on the "regularization parameter" $\delta$. We (numerically) find that $\lim _{\delta \rightarrow 0} \hat{u}(\cdot ; \delta)=\hat{u}(\cdot, 0)$ where $\hat{u}(\cdot, 0)$ is a CSS of the formal limit system, and moreover, that the associated CPs also converge (uniformly in $x$ and $t$ ) for $\delta \rightarrow 0$ to the limit CP. We also studied the dependence of CSSs and CPs on other parameters such as $c$ as we did for $f=f_{\text {lin }}$ in Figure 2. This dependence is as expected (similar to Figure 2), and thus we refrain from presenting these results in detail, and only note that $f_{\text {bi }}$ yields similar comparative statics results with respect to the parameters $(\alpha, p, \gamma, c)$ as $f_{\text {lin }}$ and $f_{\log }$.

Remark 1. In the derivation of (21) we assume the differentiability of $h$ in $v$ and $k$, and thus we need $v(0, t)>0$ and $k(t)>0$ for all $t \geq 0$. On the other hand, given (21e), we may eliminate $k$ from (21a), (21b), (21c), and (21d) and find that $h(v, k)$ $=v^{\alpha} k^{1-\alpha}=\tilde{k}(\lambda)^{1-\alpha} v$ such that

$$
\partial_{v} h=\widetilde{k}(\lambda)^{1-\alpha}
$$

exists also for $v=0$. This will now be relevant for $f=f_{\mathrm{bi}}$ when we let the small regularization parameter $\delta>0$ go to 0 . We find a branch of CSSs $\hat{u}(\cdot ; \delta)$ with monotonously increasing $0<\hat{v}(\cdot ; \delta) \approx \delta$, and monotonously decreasing $\hat{\lambda}(\cdot ; \delta)>0$. Moreover, for $\delta \rightarrow 0$, we find that $\hat{u}(\cdot ; \delta) \rightarrow \hat{u}_{0}(\cdot)$, with $\hat{v}_{0} \equiv 0$ and $\hat{\lambda}_{0}$ the solution of $(21)$ obtained from setting $\delta=0$ and using (45), and that, given some initial state $v_{0}$ (with low stocks), the CPs to $\hat{u}(\cdot, \delta)$ converge to the CP to $\hat{u}_{0}(\cdot)$ as well. This justifies using the CS (21) with (45) also for $\delta=0$, although a direct derivation of (21) for this case fails due to the nondifferentiability of $h$ at $(v, k)=(0,0)$.

\footnotetext{
${ }^{3}$ In these and similar plots to follow, " $11 /$ pt 13" denotes the target CSS, following the pde2path style branch/pointnumber for solution labeling. In the surface plots $(x, t) \mapsto v(x, t)$ we only use a reduction of the computational grid for plotting, that is, typically every third or fourth point in the $x$ and $t$ directions. The computation of the CPs uses adaptive mesh-refinement in $t$, which usually occurs at the fast transitions at the start of the CPs.
} 
(a) Continuation diagram of CSSs in $\rho$
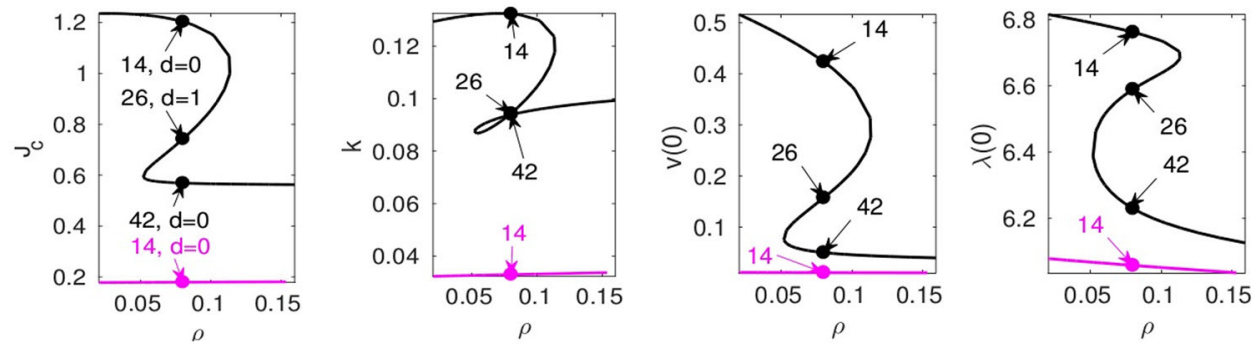

(b) Example CSS
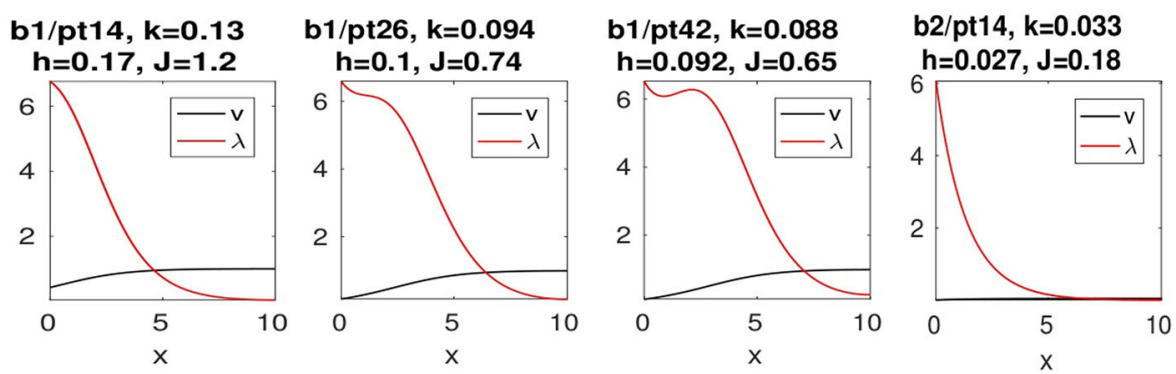

F I G U RE 4 CSSs for $f=f_{\text {bi }}$, parameters from (44), $\delta=0.05$. (a) Continuation in $\rho$ of two branches of CSSs, leading to multiple CSSs (at fixed parameter values). $d=0$ (saddle points) and $d=1$ refers to the defects of the CSS on the respective (sections of the) branches. (b) Example CSS from the points marked in (a), b1 = black branch, $\mathrm{b} 2=$ magenta branch, [...] cf. footnote 3 for the naming convention

\subsection{1 $\quad$ The case $\delta>0$}

Figure 4 illustrates that for the choice (44) and $\delta=0.05$ we have up to four CSSs for, for example, $\rho \in\left(\rho_{0}, \rho_{1}\right)$, where the left and right folds of the black branch are at $\rho_{0} \approx 0.051$ and $\rho_{1} \approx 0.11$, respectively. The continuation of this branch starts in the upper left with a solution obtained from an initial guess of the form $v(x)=0.8+0.1 x / l$ and $\lambda(x)=1$. The CSSs on the first part (till the fold) have defect $d=0$, on the middle part, we have $d=1$, and after the second fold $d=0$ again. Here the first (second and third) parts correspond to solutions of "black" ("blue") type in Figure 1d. Additionally we have the magenta branch obtained from an initial guess of the form $v(x)=\delta / 2, \lambda(x)=1$, with $d=0$ throughout. The example solution plots in (b) show four CSSs at $\rho=0.06$ from (a). If we continue an "upper solution" such as $\mathrm{b} 1 / \mathrm{pt} 14$ in the costs $c$, then we obtain a very similar behavior as in Figure 2 for $f=f_{\text {lin }}$ and for $f=f_{\log }$. Clearly, this can be expected from the phase portrait in Figure 1c, as northwest of and close to the fixed point $\left(v, v^{\prime}\right)=(1,0)$, all three phase portraits are similar. Continuation in $c$ of solutions from the other branches, including the magenta branch, behave accordingly.

However, in contrast to the case of $f_{\text {lin }}$ and $f_{\text {log }}$, the upper solutions are not globally stable, that is they cannot be reached from arbitrary initial states $v_{0}$. For illustration, we fix $\rho=0.06$ and thus have $\mathrm{b} 1 / \mathrm{pt} 14, \mathrm{~b} 1 / \mathrm{pt} 42$, and b2/pt14 (magenta CSS) as possible target CSSs, while $\mathrm{b} 1 / \mathrm{pt} 26$ cannot be a target CSS as it has defect $d=1>0$. It then depends on the initial state $v_{0}$, which CSS can be reached by a CP starting in $v_{0}$, and which of these CPs is optimal. In Figure $5 \mathrm{a}, \mathrm{b}$, we start with $v_{0}$ from the defective CSS b1/pt26. From this $v_{0}$ we can reach both, $\mathrm{b} 1 / \mathrm{pt} 14$, and $\mathrm{b} 1 / \mathrm{pt} 42$, and both are locally stable OSSs. To reach b1/pt 14 from $v_{0}$, we need to temporarily decrease $k$ to almost 0 , while $k$ is almost constant on the $\mathrm{CP}$ to $\mathrm{b} 1 / \mathrm{pt} 42$. 
(a) from $v_{0}$ from $\mathrm{b} 1 / \mathrm{pt} 26$ to $\mathrm{b} 1 / \mathrm{pt} 14$
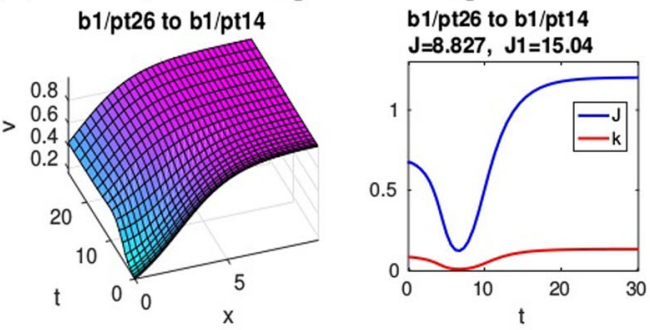

(c) from $v_{0}=\max (0.2+x / 10,1)$ to $\mathrm{b} 1 / \mathrm{pt} 14$
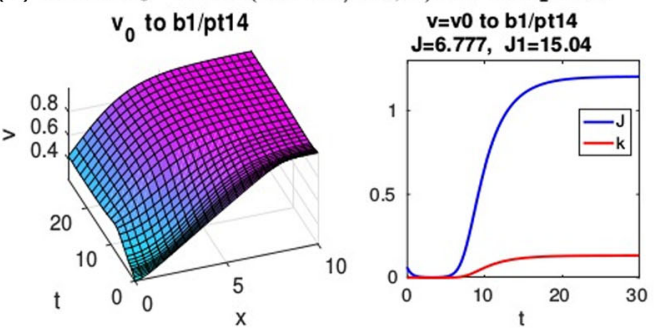

(e) from $v=0.51$ to $\mathrm{b} 2 / \mathrm{pt} 14$
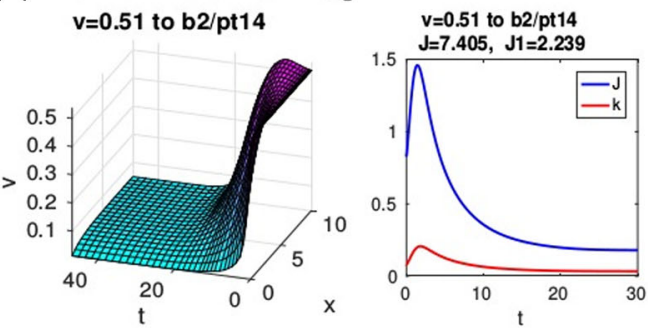

(b) from $v_{0}$ from $\mathrm{b} 1 / \mathrm{pt} 26$ to $\mathrm{b} 1 / \mathrm{pt} 42$
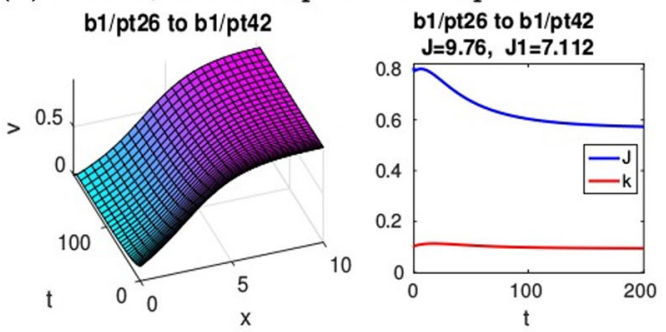

(d) from $v_{0} \equiv 0.4$ to $\mathrm{b} 2 / \mathrm{pt} 14$
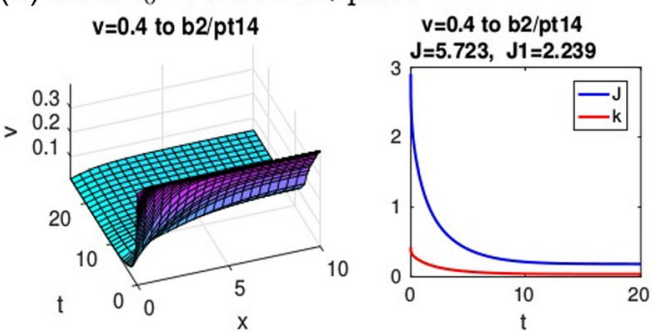

(f) from $v=0.51$ to $\mathrm{b} 1 / \mathrm{pt} 14$
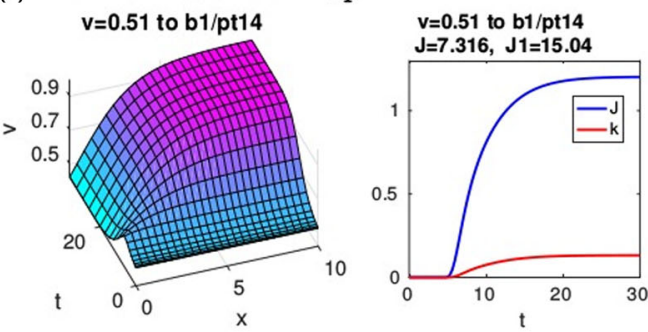

F I G U RE 5 Example CPs for $f=f_{\text {bi }}$, parameters from (44), $\delta=0.05$. In the time-series plots, $J$ and $J 1$ give the values of the CP and the target CSS, respectively. In (a) and (b), we use the same initial state $v_{0}$ from the defective CSS $\mathrm{b} 1$ /pt 26, but connect to the two different saddle-point CSSs on the black branch. The CP in (b) gives a somewhat higher $J$, although the target in (a) has a higher $J 1$. (c) Example of a CP with "waiting time" for the stock to first increase at $x=0$. (d) Example CP for the "subthreshold" initial state $v \equiv 0.4<\beta=0.5$, connecting to the magenta CSS b2/pt14. (e,f) Two CPs for the constant "intermediate" initial state $v \equiv 0.51$

Therefore, even though the CSS b1/pt42 has a significantly lower value $\left(J_{1}=7.11\right)$ than the CSS b $1 /$ pt $14\left(J_{1}=15.04\right)$, in this case, it is preferable to control the system toward b1/pt 42 : For the CP to $\mathrm{b} 1 / \mathrm{pt} 42$ we obtain $J \approx 9.76$, while the CP to $\mathrm{b} 1 / \mathrm{pt} 14$ only yields $J \approx 8.83$. A $\mathrm{CP}$ to the low-stock CSS b2/pt 14 does not exist.

In Figure 5c, we give an illustration of the possible "waiting time" phenomenon associated with CPs going to a CSS with a large stock, when the initial stock at $x=0$ is low while there is sufficient stock in the rest of the domain. Here we choose $v_{0}=\max (0.2+x / 10,1)$. A CP to $\mathrm{b} 1 / \mathrm{pt} 14$ exists and has $k(t) \approx 0$ until at $t \approx 8$ the diffusion has led to a sufficient growth of the stock at $x=0$. Nevertheless, for this $v_{0}$ again, the path to $\mathrm{b} 1 / \mathrm{pt} 42$ (not shown) is dominant as it yields $J \approx 8.95$.

Due to the maximum principle for diffusion and the fact that $f(v)<0$ for $0 \leq \delta<v<\beta$, for all $v_{0}$ with $0<v_{0}(x) \leq \beta$ for all $x$, the stock cannot increase beyond $\beta$ at any point. Consequently, the only target CSSs that can be reached are those from the magenta branch. In Figure $5 \mathrm{~d}$, we consider $v_{0} \equiv 0.4$ as an example. Here, the optimal strategy is to start with a large 
harvesting effort $k$ which then monotonously decreases to that of the CSS. More generally, motivated by the bistability of $f_{\mathrm{bi}}$, we introduce the set of "subthreshold" initial states, which means that only a CP toward a low-stock CSS from the magenta branch exists, and the set of "superthreshold" initial states, for which only CPs toward the high-stock CSSs exist, where one could further distinguish between the analogs of b1/pt14 and b1/pt42. Initial states for which CPs toward both, low-stock and high-stock CSSs exist are then called "intermediate." In Figure $5 \mathrm{e}$,f we consider such an intermediate case, namely $v_{0} \equiv 0.51$. Here, CPs to b2/pt 14 and to $\mathrm{b} 1 / \mathrm{p} 14$ (but not to $\mathrm{b} 1 / \mathrm{pt} 42$ ) exist. The resulting profit $J \approx 7.405$ is (slightly) higher by going to the low-stock CSS (e) than by going to the high-stock CSS (f) with $J \approx 7.316$, as, interestingly, the latter is again associated with a waiting time ( $k$ almost 0$)$, during which the stock increases uniformly.

A constant $v_{0}>v_{0}^{0} \approx 0.52$ turns out to be superthreshold. Of course, a precise characterization of the subthreshold, superthreshold, and intermediate sets would be tantamount to the computation of the "basins of attraction" (Grass et al., 2008, p237ff) of the analogs of $\mathrm{b} 2 / \mathrm{pt} 14$, and b1/pt14 and b1/pt42 at a given set of parameters, which is not feasible for the present infinite dimensional PDE-constrained problem, or even for the high-dimensional ODE problem obtained by spatial discretization. However, our simulations show that in the class of constant $v_{0}$, the superthreshold is only slightly larger than $\beta$, and that, generally speaking, the intermediate set is rather small.

The "upper" CSSs (with large stocks at $x=0$, e.g., b1 pt 14 ) only exist up to $\rho_{1} \approx 0.11$. For higher discount rates (corresponding to more myopic fishers), only the lower black and the magenta CSSs exist, but the (rough) characterization of subthreshold, intermediate, and superthreshold initial states, and the associated CPs, are similar. Thus, so far, this example illustrates the following:

1. To get an idea about possible optimal paths, it is crucial to first have a thorough understanding of the multiple CSSs.

2. Given an initial state $v_{0}$ for which CPs to different CSSs exist (intermediate case), we need to compare the values of the CPs (not just of the target CSSs) to decide which CP is optimal.

3. Constant $v_{0}$ only slightly larger than $\beta$ are superthreshold. Further examples for superthreshold $v_{0}$ are $v_{0}$ from b1/pt26 and $v_{0}$ from Figure $5 \mathrm{c}$, where in both cases a CP to b2/pt 14 does not exist; roughly speaking, most $v_{0}$ with $v_{0}(x)$ small at small $x$ but $v_{0}(x)$ large at more distant locations are superthreshold. This shows that the boundary control is rather weak in the following sense. For a distributed control (without control constraints) we would expect that a CP to a low-stock CSS exists for all initial states $v_{0}$ (even though this $\mathrm{CP}$ might not be optimal). For the boundary harvesting, such a CP often does not exist; the bulk of fish is safe on the right, and is not sufficiently strongly "pulled over" by diffusion, and is thus protected.

\subsubsection{Low stocks and the limit $\delta \rightarrow 0$}

The magenta branch from Figure 4 exists for all (small) $\delta>0$. Moreover, via (45), we may a posteriori use the CS (21) also for $\delta=0$. Doing so, we can analytically compute the analog of the magenta branch for $\delta=0$. We have $\hat{v} \equiv 0$, and the ODE for $\lambda$ becomes

$$
D \lambda^{\prime \prime}=\left(\rho-f^{\prime}(0)\right) \lambda,
$$


(a) $\hat{v}(x ; \delta), \hat{\lambda}(x ; \delta), \delta=0.05,0.01,0$

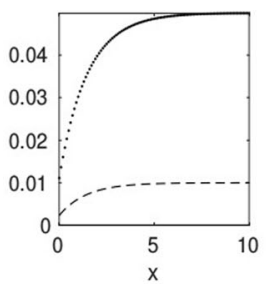

(b) $\lambda(0 ; \delta)$

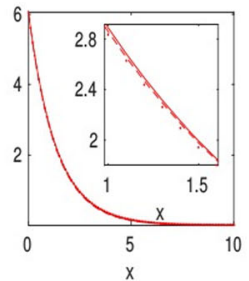

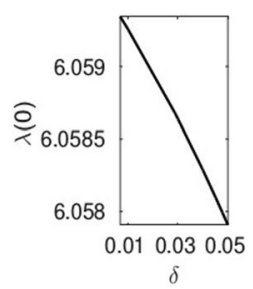

(c) $J, k$ on $\mathrm{CPs}$

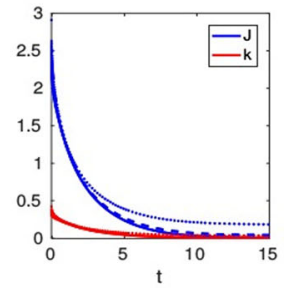

FIG URE 6 (a,b) Continuation of the CSS b $2 / \mathrm{pt} 14$ from Figure 4 in $\delta$, yielding a branch $\delta \mapsto \hat{u}(\cdot, \delta)$. (a) Spatial dependence of CSSs, $\delta=0.05$ (dots), $\delta=0.01$ (dashed), and $\delta=0$ (full lines), with a zoom inset for $\hat{\lambda}$. (b) $\lambda(0 ; \delta)$ as a function of $\delta$, with $\lim \lambda(0 ; \delta)=\lambda(0 ; 0)$ with $\lambda(0 ; 0)$ from (48). (c) $J$ and $k$ on CPs from $v_{0} \equiv 0.4$ to the CSSs from (a), $\delta$ and line types as in (a)

where $\left.f^{\prime}(0)\right|_{\delta=0}=-\beta$, with the general solution

$$
\lambda(x)=c_{1} \mathrm{e}^{\nu x}+c_{2} \mathrm{e}^{-v x}, \quad c_{1}, c_{2} \in \mathbb{R}, \quad \nu=\sqrt{(\rho+\beta) / D} .
$$

The BC $\lambda^{\prime}(l)=0$ yields $c_{2}=\mathrm{e}^{2 v l} c_{1}$, and the BC $-D \lambda^{\prime}(0)=(p-\gamma \lambda(0)) \widetilde{k}(\lambda)^{1-\alpha}$ (using (45)) then yields the algebraic equation

$$
D \nu\left(\mathrm{e}^{2 \nu l}-1\right) c_{1}=\left(p-\gamma c_{1}\left(1+\mathrm{e}^{2 \nu l}\right)\right) \tilde{k}\left(c_{1}\left(1+\mathrm{e}^{2 \nu l}\right)\right)^{1-\alpha},
$$

which can easily be solved numerically for $c_{1}$ using a Newton method with initial guess $c_{1}=0$. The solution then can be cross-checked with the numerical pde2path solution of (21) for $\hat{\lambda}(x ; 0)$, and $\hat{v}(x ; 0) \equiv 0$, yielding a perfect match.

As the CS for $\delta=0$ is only obtained formally, in Figure 6 we illustrate the (numerical) convergence of the solutions for $\delta>0$ to $\hat{u}(\cdot, 0)$ as $\delta \rightarrow 0$. For this, we continue the CSS from b2/pt 14 in $\delta$, and in Figure 6a, we display $\hat{v}(\cdot ; \delta)$ and $\hat{\lambda}(\cdot ; \delta)$ for $\delta=0.05, \delta=0.01$, and $\delta=0$. The plots of $\hat{\lambda}(\cdot, \delta)$ show that $\hat{\lambda}$ very weakly depends on $\delta$, and the differences are only visible in the zoom. Figure $6 \mathrm{~b}$ illustrates the convergence of $\lambda(0 ; \delta)$, while all other quantities such as $v(0 ; \delta), k(\delta)$ converge to 0 for $\delta \rightarrow 0$. Figure 6c illustrates the convergence of the CPs for fixed $v_{0} \equiv 0.4$ (as in Figure 4a) for $\delta \rightarrow 0$ to the CP $t \mapsto u^{(0)}(t)$ obtained for the formal limit CS ( $\delta=0$, full lines). Although we have no rigorous proof, this strongly suggests that $k^{(0)}(t)$ from $u^{(0)}(t)$ is the OC for $\delta=0$ for such "subthreshold" initial states, for which, as expected, the harvesting effort decreases monotonically to 0 . The CPs connecting to the upper black branch from Figure 4 are hardly affected by changing $\delta$, and thus we refrain from presenting these results.

\section{5 | RESULTS FOR THE PREDATOR-PREY CASE}

To apply the formalism from Section 3.1 to the vector-valued problem (15), we introduce the costates $\lambda_{1,2}$ and the Lagrangian:

$$
L(v, \lambda, k)=\int_{0}^{\infty} \mathrm{e}^{-\rho t}\left\{J_{c}-\int_{\Omega}\left\langle\lambda, \partial_{t} v+G_{1}(v)\right\rangle \mathrm{d} x\right\} \mathrm{d} t,
$$


where $\langle u, v\rangle=\sum_{i=1}^{2} u_{i} v_{i}$ is the standard inner product in $\mathbb{R}^{2}$. Integration by parts in $x$ and $t$ with the transversality condition

$$
\operatorname{lime}_{t \rightarrow \infty} \mathrm{e}^{-\rho t} \int_{\Omega}\langle\lambda(x, t) v(x, t)\rangle \mathrm{d} x=0
$$

now yields

$$
\begin{aligned}
L(v, \lambda, k)= & \int_{\Omega}\langle\lambda(0, \cdot), v(0, \cdot)\rangle \mathrm{d} x+\int_{0}^{\infty} \mathrm{e}^{-\rho t}\left\{\left.\left[J_{c}-\langle\lambda, g\rangle-\left\langle D \partial_{n} \lambda, v\right\rangle\right]\right|_{x=0}-\left.\left\langle D \partial_{n} \lambda, v\right\rangle\right|_{x=l}\right. \\
& \left.-\int_{\Omega}\left\langle\rho \lambda-\partial_{t} \lambda-D \Delta \lambda, v\right\rangle-\langle\lambda, f(v)\rangle \mathrm{d} x\right\} \mathrm{d} t .
\end{aligned}
$$

Then $\partial_{v} L=0$ yields the evolution and the BCs of the costates (combining with (10), to have it all together):

$$
\left.\begin{array}{c}
\left.\begin{array}{c}
\partial_{t} v=D \Delta v+f(v), \\
\partial_{t} \lambda=\rho \lambda-D \Delta \lambda-\left(\partial_{v} f(v)\right)^{T} \lambda
\end{array}\right\} \text { in } \Omega=(0, l), \\
D \partial_{n} v+g=0 \\
D \partial_{n} \lambda+\partial_{v} g(v) \lambda-\partial_{v} J_{c}=0
\end{array}\right\} \text { on the left boundary } x=0,
$$

and $\partial_{k} L=0$ yields

$$
k_{j}=\left(\frac{\left(1-\alpha_{j}\right)^{2}\left(p_{j}-\gamma_{j} \lambda_{j}\right)}{c_{j}}\right)^{1 / \alpha_{j}} v_{j}, \quad j=1,2 .
$$

As above, we first compute CSSs, that is, we start with the stationary version $G(u)=0$, $u=(v, \lambda)$ of $(50)$, on $\Omega=(0,20)$. We choose the base parameter set:

$$
\left(\beta, d_{1}, d_{2}, \gamma_{1}, \gamma_{2}, \rho, \alpha_{1}, \alpha_{2}, p_{1}, p_{2}\right)=(0.6,1,10,0.1,0.1,0.03,0.4,0.4,20,10),
$$

and consider the costs $\left(c_{1}, c_{2}\right)$ as our continuation parameters, starting with $c_{1}=c_{2}=0.1$. By (52), we assume that the predators move faster than the prey, $d_{2}=10>1=d_{1}$, and that the market price of the prey exceeds the price of the predator, $p_{1}=20>10=p_{2}$, so that, disregarding the interaction of both species, the fisher is interested in catching the prey rather than the predator. Yet, in view of the interaction of the species, the fisher may consider catching the predator as well to "protect" the prey from the predator.

To find CSSs we use initial guesses of the form

$$
v_{1}=1, \quad v_{2}=\delta(1-\beta), \quad \lambda_{1}=50+(1-s x / l), \quad \lambda_{2}=10+(1-s x / l),
$$


(a) continuation in $c_{1}, J, v, \lambda, k, h$ at $x=0$
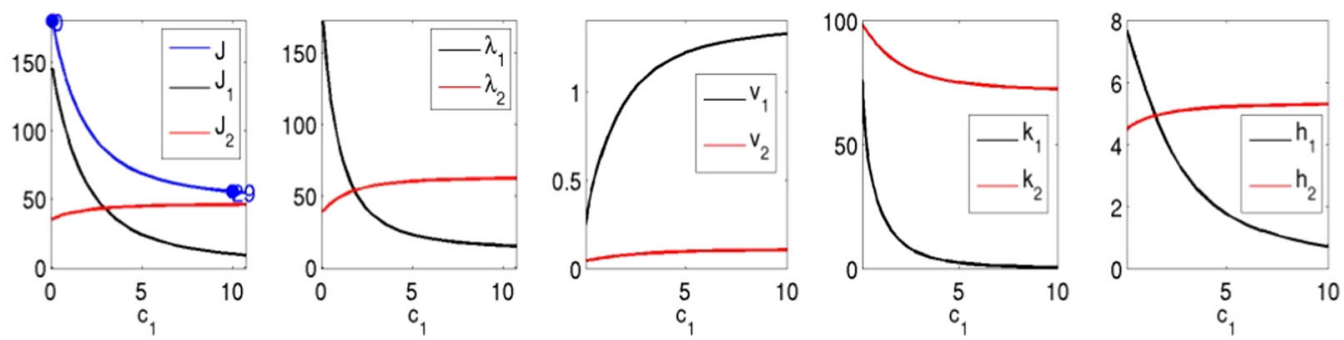

(b) continuation in $c_{2}, J, v, \lambda, k, h$ at $x=0$
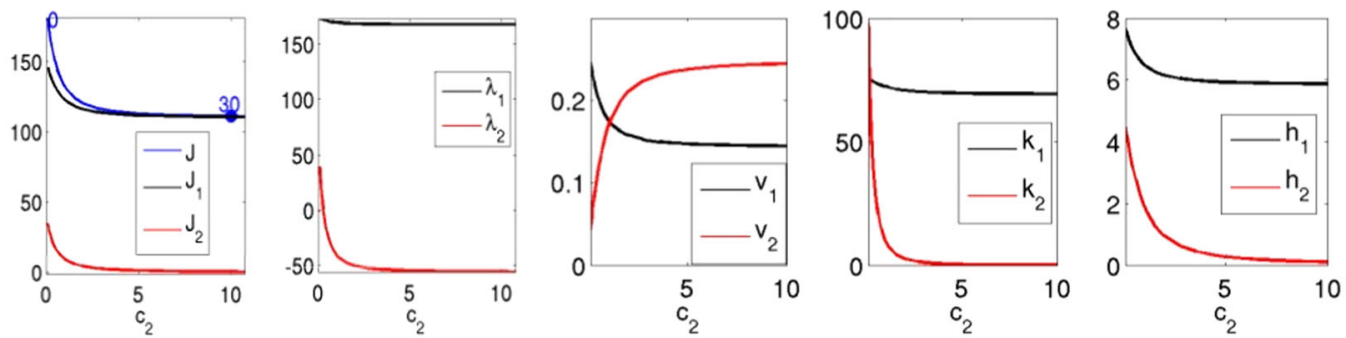

(c) CSS at $c=(0.1,0.1), c=(10,0.1)$ and $c=(0.1,10)$
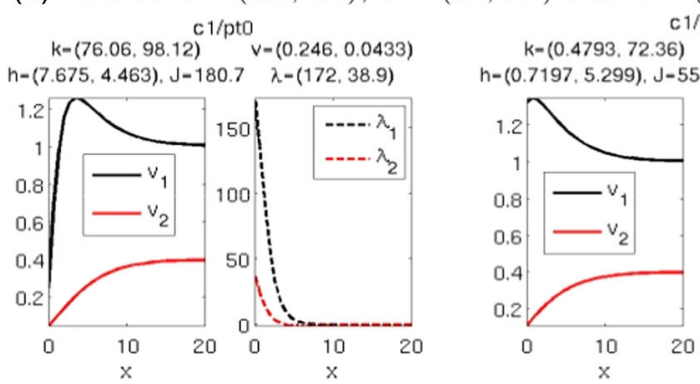

c $1 /$ pt29
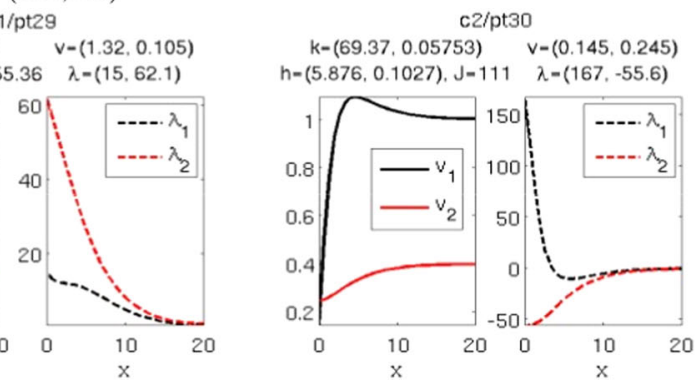

FIG URE 7 (a,b) Continuation diagrams in $c_{1}$ (costs for prey fishing) and $c_{2}$ (costs for predator fishing); $J_{j}=p_{j} h_{j}-c_{j} k_{j}, J=J_{1}+J_{2}$. For graphical reasons, we restrict to $c_{j} \in[0.1,10]$ (except for the plots of $J$ ) but all quantities continue as expected for $c_{j} \in[10,20]$. (c) Example CSS plots, where $k=\left(k_{1}, k_{2}\right)$ and $h=\left(h_{1}, h_{2}\right)$ give the control and the harvest, and $v=\left(v_{1}, v_{2}\right)$ and $\lambda=\left(\lambda_{1}, \lambda_{2}\right)$ are the values of the states and the co-states at the left boundary $x=0$.

with parameters $\delta \in(0,1)$ and $s$ close to 1 , and some variations of $(53)$. But if an initial guess of this form yields convergence to a CSS, then (for the base parameters (52) this convergence always leads to the same CSS. Note that a graphical phase plane analysis similar to Section 2.2 is no longer possible, and thus it is not clear how many CSSs may exist for (50). However, given that for $d_{1,2}$ sufficiently large and zero-flux $\mathrm{BCs} V^{*}$ is the unique steady state of the Lotka-Volterra system (10), it appears reasonable to expect that for the given parameters, the system (50) has a unique CSS, as we assume rather large values for the diffusion parameters, $\left(d_{1}, d_{2}\right)=(1,10)$, relative to the size of the domain.

Figure 7 depicts the CSSs and their dependence on the cost parameters $\left(c_{1}, c_{2}\right){ }^{4}$ Figure $7 \mathrm{a}, \mathrm{b}$ shows relevant quantities at the left boundary as functions of $c_{1}$ and $c_{2}$, respectively, while 
Figure $7 \mathrm{c}$ shows the spatial shape of the CSSs for selected values of $\left(c_{1}, c_{2}\right)$. As expected, an increase in $c_{i}(i=1,2)$ leads to a reduction in effort $k_{i}$ and the associated harvest $h_{i}$, and thus leads to a recovery of the stock $v_{i}$. In addition, an increase in $c_{i}$ also imposes an indirect effect on $v_{3-i}$ resulting from the interaction between both species. With respect to an increase in the effort $\operatorname{cost} c_{1}$, there is no direct effect of $c_{1}$ on the fishing effort $k_{2}$ as its cost as well as the market price $p_{2}$ are unaffected. However, as an increase in $c_{1}$ results in less fishing effort $k_{1}$ and thus in a higher stock $v_{1}$, the living conditions of the predator species improve. Accordingly, the stock $v_{2}$ tends to increase, but as $v_{2}$ and $k_{2}$ are complements in the fishing technology, effort $k_{2}$ can be reduced with the catch $h_{2}$ still going up. Naturally, with a higher $\operatorname{cost} c_{1}$ the value of the prey $\lambda_{1}$ falls, but the indirect effect makes the value of the predator $\lambda_{2}$ go up. The direct and the indirect effect of an increase in $c_{1}$ are also reflected in the profit terms. As expected, $J_{1}$ is a decreasing function of $c_{1}$, but the induced interaction effects between both species make $J_{2}$ to increase with $c_{1}$. As the direct effect dominates, total profit $J$ falls with higher cost.

Due to the biologically asymmetric situation of both species, an increase in $c_{2}$ has somewhat different indirect effects. In this case, a higher $c_{2}$ leads to a reduction in effort $k_{2}$ and thus to an increase in the stock $v_{2}$. But with an increase of the predators the prey become more threatened, rendering this population to decline, and thus the fishing effort $k_{1}$ is reduced. In this way, as the increase in $c_{2}$ acts as protection of the predator against being fished, the growth of that population exerts a negative effect on the prospects of fishing for the fisher. Accordingly, with the predator population becoming sufficiently large, the value of a unit of this species becomes negative. This explains why $\lambda_{2}$ is decreasing in $c_{2}$ and why it becomes (quickly) negative as the stock $v_{2}$ rises.

The qualitative structure of the spatial distribution of both species (and of the associated shadow prices) is quite robust with respect to changes in the $\operatorname{costs} c_{1}, c_{2}$. Inspecting $c_{1}, c_{2}$, and $c_{3}$ of Figure 7, we infer that by catching the predator species, the fisher makes sure that its stock is kept low at the coast (left boundary) so as to safeguard the prey there. In fact, the stock of the prey reaches its maximum close to the coast, but harvesting at the coast causes the stock to decrease drastically there (unless $c_{1}$ is very high, see $c_{2}$ of Figure 7). In any case, fish located close to the coast is more worthy than at more distant locations, where it is inaccessible for the fisher, that is, $\lambda_{1}$ and $\lambda_{2}$ are both decreasing in $x$. There is one exception, though: When, as explained above, $c_{2}$ is sufficiently large, such that it is very expensive to catch the predator species, this stock may swell until it interferes with the prospects of the fisher to catch the prey. In this case, the value of the predator is negative, $\lambda_{2}<0$, and because the damage caused by the predator species is the larger the closer it gets to the shore, $\lambda_{2}$ has its minimum directly at the shore.

In Figure 8, we illustrate the transition dynamics from the unique steady state (11) (with no fishing) to the CSS. Setting $\left(c_{1}, c_{2}\right)=(0.1,0.1)$ in Figure $8 \mathrm{a}$ and $\left(c_{1}, c_{2}\right)=(20,0.1)$ in Figure $8 \mathrm{~b}$, these figures can directly be compared with $c_{1}$ and $c_{2}$ of Figure 7 , respectively. When the fishing cost of both species are low, $\left(c_{1}, c_{2}\right)=(0.1,0.1)$, the transition to the CSS is accomplished by extensive fishing of both species at an initial phase, with fishing intensities decreasing from high toward low values. Thus, there is a some initial overfishing, followed by a recovery phase during which the stocks of the CSS are reached from below, and during which the fisher increases the fishing intensity to that of the $\mathrm{CSS}^{5}$ If fishing the prey is very costly, $\left(c_{1}, c_{2}\right)=(10,0.1)$, the CSS is characterized by a low effort level $k_{1}$ and hence by a high stock of

${ }^{5}$ The initial transition is rather fast, and thus we use logarithmic scales in the time-series of the values at the left boundary. 
(a) CP from $V^{*}$ to the CSS at $\left(c_{1}, c_{2}\right)=(0.1,0.1)$

Eq to $1 / p t 0$
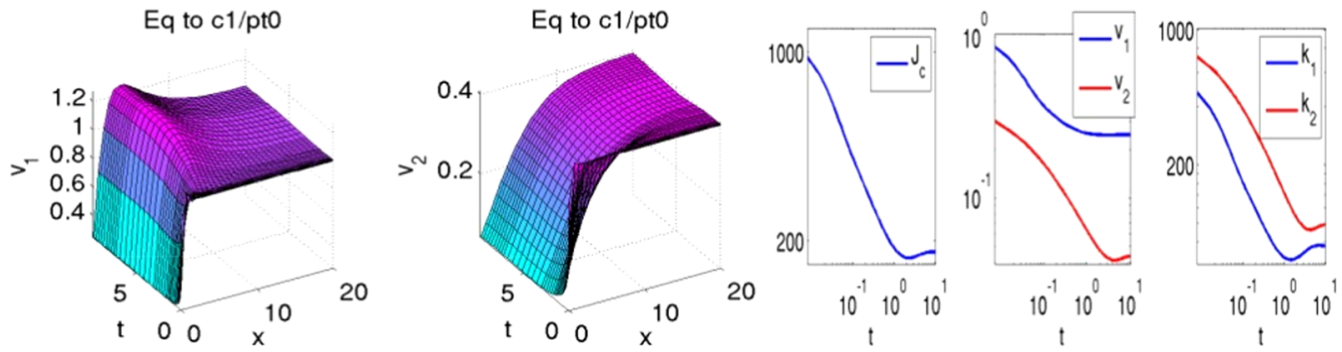

(b) CP from $V^{*}$ to the CSS at $\left(c_{1}, c_{2}\right)=(10,0.1)$

Eq to $c 1 / p t 29$

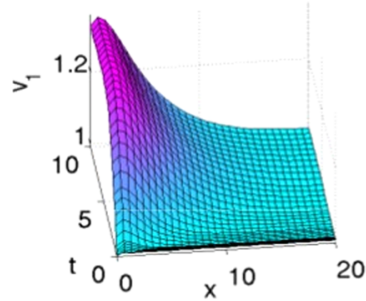

Eq to $c 1 / p t 29$
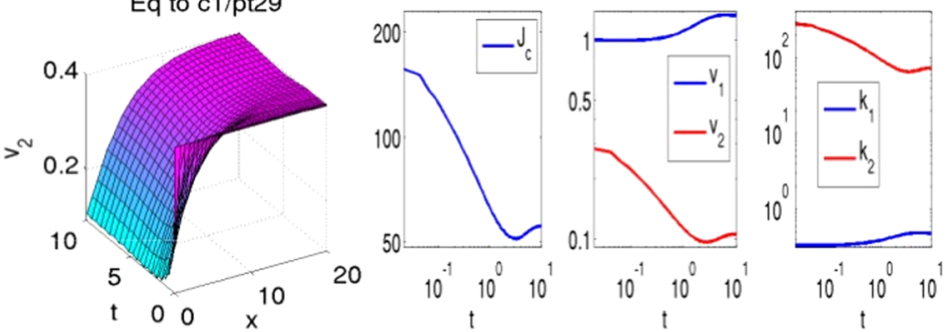

(c) CP from $V^{*}$ to the CSS at $\left(c_{1}, c_{2}\right)=(0.1,10)$
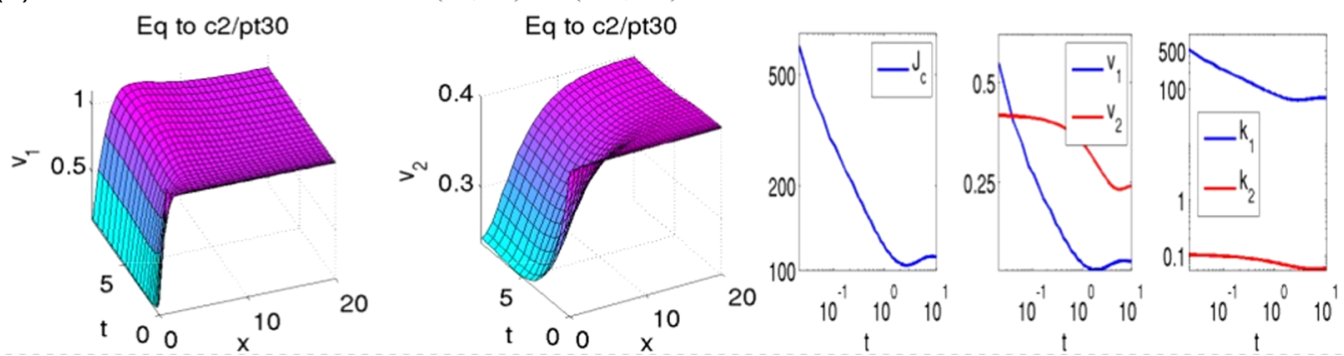

F I G URE 8 Canonical paths starting from the spatially homogeneous steady state $V^{*}$ of(10)

the prey, comparing Figure $7\left(c_{2}\right)$ and $8 \mathrm{~b}$. Similarly, along the CP leading to the CSS, the main harvest is on the predator, with fishing effort being reduced over time, leading to the gradual increase of the prey at the left boundary-and eventually on the complete domain. However, some harvesting activity on the prey still takes place. Finally, for $\left(c_{1}, c_{2}\right)=(0.1,10)$, the roles are basically reversed, subject to the indirect effects resulting from the implicit protection of the predator species by a high effort cost $c_{2}$, as explained above.

\section{6 | DISCUSSION AND EXTENSIONS}

To the best of our knowledge, in resource economics this is the first detailed numerical (and in Section 4.1.1 semianalytical) analysis of infinite time-horizon OC problems with PDE constraints and a boundary control, beyond linear-quadratic models as reviewed in Section 3.3. We have set up one-species and two-species fishery models and characterized their CSSs, which turned out to be unique globally stable OSSs, except for the bistable case in the onespecies model, for which multiple locally stable OSSs exist. Moreover, while previous studies in 
marine economics focus on the CSSs, we also compute the time-dependent CPs connecting initial states to OSSs; these describe the policies that achieve the OSS in the most profitable way.

However, as our derivation of the CSs is formal in the sense detailed in Section 3.3, the CSSs and CPs obtained must be checked a posteriori for plausibility, but with this caveat, the method seems highly effective. For the given examples, all results appear natural and intuitive, and they are robust with respect to alternative parameter specifications. The optimal policy compromises between immediate and future yield, taking into account that a higher stock left may favor future growth of the resource, and the optimal harvesting may feature "waiting times" allowing the stock to grow before fishing starts. For the bistable case, we can identify subthreshold and superthreshold initial stocks, for which only CPs to low-stock CSSs, respectively, high-stock CSSs exist. For initial states from an "intermediate" set we can go to both types of CSSs, and to determine which $\mathrm{CP}$ is optimal we need to compute the CPs and their values. This intermediate set turns out to be rather small, while the set of superthreshold initial states is rather large. This illustrates some natural limits of boundary controls compared with the distributed controls.

For the two-species Lotka-Volterra model, the asymmetric interaction between both species provides an additional incentive to catch the predator species to protect the prey for the purpose of own takeout. This asymmetry between both species carries over to the spatial distribution of the biomass of both species and their respective shadow prices: Whereas the shadow price of the prey decreases in the distance to the location of the fisher, the shadow price of the predator may be higher at more distant locations, and may increase as the harvesting cost of the prey goes up.

Keeping the above caveat about the formal derivations of the CSs in mind, our approach can be extended to more complex and realistic models. One obvious way would be to generalize the one-dimensional problems by, for instance, considering either spatially dependent coefficients (of the PDEs), or more intricate multispecies fishery models; in addition, we may consider advection to model the transport of fish by flow. A second class of generalizations would be to extend the spatial domain to two (or even three) dimensions. The spatial domain could either take the form of depth dependence of fishing at a fixed position in a river with one horizontal dimension, of a lake, or of a marine reserve in the open sea. In all these cases, we have a boundary control function defined on a given part of the boundary, rather than a scalar control.

Similar boundary control models occur in other bioeconomic circumstances. For instance, the shallow lake OC model deals with the phosphorus contamination of a lake, with the phosphate load, for instance from fertilizers, as control. Naturally, there is diffusion of the phosphorus, and thus a spatially extended model with a distributed control is studied in Grass and Uecker (2017). However, more realistically, the phosphate load should be taken as a boundary control. Finally, models as in Section 3.3.1, including boundary controls, appear in a variety of settings, see, for instance, Faggian (2004) and the references therein. It will be interesting to extend these by genuine nonlinear terms. Moreover, as $x$ in Section 3.3.1 models the age of consumers, we have a transport equation for the goodwill, one could additionally introduce a spatial coordinate $y$ for the location of consumers, which may diffuse in space, leading to a mixed 2D problem.

\section{ACKNOWLEDGMENTS}

We thank the participants of the 16th Journées Louis-André Gérard-Varet, Aix-en-Provence (2017); the Research Seminar on Environment, Resource and Climate Economics, MCC Berlin (2017); the 6th World Congress of Environmental and Resource Economists (WCERE), Gothenburg, Sweden; the 14th Viennese Conference on Optimal Control and Dynamic Games, 
TU Wien; the 6th International Workshop on Heterogeneous Dynamic Models of Economic and Population Systems, WU Vienna and, in particular, Vladimir Veliov and Charles Figuieres for valuable comments and suggestions.

\section{ORCID}

\section{Hannes Uecker (D) http://orcid.org/0000-0003-1378-5209 \\ Thorsten Upmann (D) http://orcid.org/0000-0001-7804-7982}

\section{REFERENCES}

Aniţa, S (2000). Analysis and control of age-dependent population dynamics. Dordrecht: Kluwer Academic Publishers.

Aniţa, S., Arnăutu, V., \& Capasso, V. (2011). An introduction to optimal control problems in life sciences and economics. New York: Birkhäuser/Springer.

Baker, C. M., Diele, F., Marangi, C., Martiradonna, A., \& Ragni, S. (2018). Optimal spatiotemporal effort allocation for invasive species removal incorporating a removal handling time and budget. Natural Resources Research, 31(3), 12190.

Ballestra, L. V. (2016). The spatial AK model and the Pontryagin maximum principle. Journal of Mathematical Economics, 67, 87-94.

Barucci, E., \& Gozzi, F. (2001). Technology adpotion and accumulation in a vintage capital model. Journal of Economics, 74(1), 1-38.

Behringer, S., \& Upmann, T. (2014). Optimal harvesting of a spatial renewable resource. Journal of Economic Dynamics and Control, 42, 105-120.

Beyn, W., Pampel, T., \& Semmler, W. (2001). Dynamic optimization and Skiba sets in economic examples. Optimal Control Applications and Methods, 22(5-6), 251-280.

Boucekkine, R., Camacho, C., \& Fabbri, G. (2013). Spatial dynamics and convergence: The spatial AK model. The Journal of Economic Theory, 148(6), 2719-2736.

Brock, W. A., \& Xepapadeas, A. (2008). Diffusion-induced instability and pattern formation in infinite horizon recursive optimal control. Journal of Economic Dynamics and Control, 32(9), 2745-2787.

Brock, W. A., \& Xepapadeas, A. (2010). Pattern formation, spatial externalities and regulation in coupled economic-ecological systems. Journal of Environmental Economics and Management, 59(2), 149-164.

Casas, E., Raymond, J.-P., \& Zidani, H. (2000). Pontryaginas principle for local solutions of control problems with mixed control-state constraints. SIAM Journal on Control and Optimization, 39(4), 1182-1203.

Casas, E., Ryll, C., \& Tröltzsch, F. (2018). Optimal control of a class of reaction-diffusion systems. Computational Optimization and Applications, 70(3), 677-707.

Casas, E., \& Tröltzsch, F. (2015). Second order optimality conditions and their role in PDE control. Jahresbericht der Deutschen Mathematiker Vereinigung, 117(1), 3-44.

Clark, C. W. (2010). Mathematical bioeconomics (3rd ed.). New Jersey: John Wiley \& Sons.

Conrad, J. M. (2010). Resource economics (2nd ed.). Cambridge: Cambridge University Press.

Conrad, J. M., \& Clark, C. W. (1987). Natural resource economics: Notes and problems. Cambridge, New York: Cambridge University Press.

Da Lara, M., \& Doyen, L. (2008). Sustainable managment of natural resources: Mathematical models and methods. Berlin \& Heidelberg: Springer.

Ding, W., \& Lenhart, S. (2009). Optimal harvesting of a spatially explicit fishery model. Natural Resource Modeling, 22(2), 173-211.

Faggian, S. (2004). Boundary-control problems with convex cost and dynamic programming in infinite dimension. I. The maximum principle. Differential Integral Equations, 17(9-10), 1149-1174.

Faggian, S., \& Grosset, L. (2013). Optimal advertising strategies with age-structured goodwill. Mathematical Methods in Operations Research, 78(2), 259-284. 
Feichtinger, G., T. G., \& Veliov, V. (2003). Optimality conditions for age-structured control systems. Journal of Mathematical Analysis and Applications, 288(1), 47-68.

Fister, K. R. (1997). Optimal control of harvesting in a predator-prey parabolic system. Houston Journal of Mathematics, 23(2), 341-355.

Fister, K. R. (2001). Optimal control of harvesting coupled with boundary control in a predator-prey system, Applicable Analysis, 77(1-2), 11-28.

Fister, K. R., \& Lenhart, S. (2006). Optimal harvesting in an age-structured predator-prey model. Applied Mathematics and Optimization, 54(1), 1-15.

Fogarty, M. J., \& Murawski, S. A. (2004). Do marine protected areas really work? Oceanus Magazine, 43(2), 1-3.

Grass, D., Caulkins, J. P., Feichtinger, G., Tragler, G., \& Behrens, D. A. (2008). Optimal control of nonlinear processes: With applications in drugs, corruption, and terror. Berlin \& Heidelberg: Springer.

Grass, D., \& Uecker, H. (2017). Optimal management and spatial patterns in a distributed shallow lake model. Electronic Journal of Differential Equations, 1, 1-21.

Hastings, A. (1978). Global stability in Lotka-Volterra systems with diffusion. Journal of Mathematical Biology, 6(2), 163-168.

Hinze, M., Pinnau, R., Ulbrich, M., \& Ulbrich, S. (2009). Optimization with PDE constraints. Mathematical modelling: Theory and applications (23). Netherlands: Springer.

Kellner, J. B., Tetreault, I., Gaines, S. D., \& Nisbet, R. M. (2007). Fishing the line near marine reserves in single and multispecies fisheries. Ecological Applications, 17(4), 1039-1054.

Kelly, M. R., Jr., Xing, Y., \& Lenhart, S. (2016). Optimal fish harvesting for a population modeled by a nonlinear parabolic partial differential equation. Natural Resource Modeling, 29(1), 36-70.

Kunkel, P., \& von dem Hagen, O. (2000). Numerical solution of infinite-horizon optimal-control problems. Computational Economics, 16, 189-205.

Lenhart, S., Liang, M., \& Protopopescu, V. (1999). Optimal control of boundary habitat hostility for interacting species. Mathematical Methods in the Applied Sciences, 22(13), 1061-1077.

Lenhart, S., \& Workman, J. (2007). Optimal control applied to biological models. Boca Raton, London, New York: Chapman \& Hall/CRC.

Leung, A. W. (1995). Optimal harvesting-coefficient control of steady-state prey predator diffusive Volterra-Lotka systems. Applied Mathematics and Optimization, 31(2), 219-241.

Li, X. J., \& Yong, J. M. (1995). Optimal control theory for infinite-dimensional systems. Boston: Birkhäuser.

Lions, J.-L. (1971). Optimal control of systems governed by partial differential equations. Grundlehren der mathematischen Wissenschaften (170). Berlin \& Heidelberg: Springer-Verlag.

McCauley, D. J., Woods, P., Sullivan, B., Bergman, B., Jablonicky, C., Roan, A., \& Worm, B. (2016). Ending hide and seek at sea. Science, 351(6278), 1148-1150.

Neubert, M. G. (2003). Marine reserves and optimal harvesting. Ecology Letters, 6(9), 843-849.

Pontryagin, L. S., Boltyanskii, V. G., Gamkrelidze, R. V., \& Mishchenko, E. F. (1962). The mathematical theory of optimal processes. New York: Wiley-Interscience.

Raymond, J. P., \& Zidani, H. (1999). Pontryagin's principle for time-optimal problems. Journal of Optimization Theory and Applications, 101(2), 375-402.

Sanchirico, J. N., \& Wilen, J. E. (1999). Bioeconomics of spatial exploitation in a patchy environment. Journal of Environmental Economics and Management, 37(2), 129-150.

Serovaiskii, S. Y. (2003). Counterexamples in optimal control theory. Inverseand Ill-Posed Problems (45). De Gruyter.

Tauchnitz, N. (2015). The pontryagin maximum principle for nonlinear optimal control problems with infinite horizon. Journal of Optimization Theory and Applications, 167(1), 27-48.

Tröltzsch, F. (2009). Optimale Steuerung partieller Di erentialgleichungen: Theorie,Verfahren und Anwendungen, 2. Auage, Series: Studium, Vieweg \& Teubner, Wiesbaden.

Uecker, H. (2016). Optimal control and spatial patterns in a semi-arid grazing system. Natural Resource Modeling, 29(2), 229-258.

Uecker, H. (2017). Infinite time-horizon spatially distributed optimal control problems with pde2path-A tutorial. Available at http://www.staff.uni-oldenburg.de/hannes.uecker/pde2path/tutorials.html

Uecker, H. (2019). Hopf bifurcation and time periodic orbits with pde2path-algorithms and applications. Communications in Computational Physics, 25(3), 812-852. 
Uecker, H., Wetzel, D., \& Rademacher, J. D. M. (2014). pde2path-A matlab package for continuation and bifurcation in 2D elliptic systems. Numerical Mathematics, 7, 58-106.

Wirl, F. (1996). Pathways to Hopf bifurcation in dynamic, continuous time optimization problems. Journal of Optimization Theory and Applications, 91, 299-320.

Xepapadeas, A. (2010). The spatial dimension in environmental and resource economics. Environment and Development Economics, 15(6), 747-758.

How to cite this article: Grass D, Uecker H, Upmann T. Optimal fishery with coastal catch. Natural Resource Modeling. 2019;32:e12235. https://doi.org/10.1111/nrm.12235 